\title{
Synergistic effects of autochthonous probiotic bacterium and Mentha piperita diets in Catla catla (Hamilton, 1822) for enhanced growth and immune response
}

\author{
Anita Bhatnagar *i) and Sonal Saluja
}

\begin{abstract}
Two dietary experiments were performed to evaluate the impact of the herb Mentha piperita as a dietary supplement on Catla catla. In Experiment 1, fingerlings $(0.45-2.60 \mathrm{~g})$ were fed on diets supplemented with M. piperita at different levels (P1, P2, P3, and P4 at 2, 4, 6, and $8 \mathrm{~g} \mathrm{Kg}^{-1}$ of feed) and C1 served as the control with no peppermint supplementation. In Experiment 2, fingerlings ( $0.40-3.15 \mathrm{~g}$ ) were fed with diets containing autochthonous probiotic bacteria Bacillus coagulans (3000 colony forming unit $\mathrm{g}^{-1}$ ) along with M. piperita (PP1-PP4 at 2, 4, 6, and $8 \mathrm{~g} \mathrm{Kg}^{-1}$ of feed) and feed C2 served as control containing probiotics but no peppermint. Significantly $(P<0.05)$ high values of growth parameters, digestive enzyme activities, low reed conservation ratio, low excretion of ammonia, and orthophosphates were observed in fingerlings fed with P3 for Experiment 1 and PP3 for Experiment 2. Hematological counts, phagocytic activity, and respiratory burst activity were also enhanced in fingerlings fed with M. piperita supplementation revealing that dietary administration of peppermint at optimum level can enhance the growth, digestibility, and immunity of $C$. catla fingerlings; however, incorporation of $B$. coagulans showed better growth and immunity revealing that synergistic effects of $M$. piperita and autochthonous probiotic in C. catla diets can lead to its sustainable aquaculture.
\end{abstract}

Keywords: Autochthonous, Bacillus coagulans, Immunity, Mentha piperita, Phagocytic, Probiotic

\section{Background}

Intensification of aquaculture makes fishes more prone to outbreak of many infectious diseases. High intensity of fishes and shortfall of hygienic conditions not only diminish the fishes well-being but also increase the probability of fish diseases due to the facilitation of pathogen dispersal (Quesada, Paschoal, and Reyes, 2013). As fish demand is continuously increasing with the increasing human population, there is an immediate need for increased disease resistance, growth performance, and feed efficiency in intensified fish culture without the usage of antibiotics or chemotherapeutics. Conventionally used antibiotics, chemotherapeutics for improving fish health (Ghosh, Sinha, and Sahu, 2007), many antimicrobials, and

* Correspondence: anitabhatnagar@gmail.com

Department of Zoology, Kurukshetra University, Kurukshetra, Haryana 136119, India

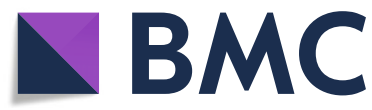

other veterinary drugs that may act as prophylactics and growth performers (Rico et al., 2013) are not safe for the environment as well as for human health, and these drugs may result in the development of a resistant strain of bacteria and virus (Seyfried, Newton, Rubert, Pedersen, and McMahon, 2010). Synthetic immunostimulants could have a good approach towards replacing these antimicrobials and chemotherapeutics but to a limited extent due to its retaining residues in the environment. So there is a drift towards the use of herbal and plant extracts to manage disease in aquaculture as these are environmentfriendly methods that have the potential to improve aquatic prosperity. Among plants, Mentha piperita (peppermint) is a hybrid mint produced by the cross of water mint and spearmint and also one of the most important medicinal plants produced all over the world. Mentha sp. have some significant properties like anticancer and anti-inflammatory (Blumenthal, 1998) and many

(c) The Author(s). 2019 Open Access This article is distributed under the terms of the Creative Commons Attribution 4.0 International License (http://creativecommons.org/licenses/by/4.0/), which permits unrestricted use, distribution, and reproduction in any medium, provided you give appropriate credit to the original author(s) and the source, provide a link to the Creative Commons license, and indicate if changes were made. The Creative Commons Public Domain Dedication waiver (http://creativecommons.org/publicdomain/zero/1.0/) applies to the data made available in this article, unless otherwise stated. 
remarkable impacts on hematological and immunological responses (Nobakht and Mehmannavaz, 2010). Furthermore, many reported literature have proven that peppermint acts as an important feed additive for increasing growth performance and immune response as well as disease resistance for different fishes.

Probiotics have also gained appreciation as an approach towards combating and controlling diseases in aquaculture. According to FAO/WHO (2001), probiotics are live microbes which when administered in suitable extent confers health assistance on the host. Several species of different bacteria such as Bacillus in Catla catla and Cirrhinus mrigala (Bandyopadhyay, 2004), Lactobacillus (Balcazar et al., 2007), and Aeromonas and Pseudomonas (Nayak and Mukherjee, 2011) in different carp species have been isolated and purified from the fish gut and integrated in formulated diet to study their effects on growth and immunity. In earlier studies, in our laboratory, attempts have been made to use commercial probiotics in fish feed (Sushma, 2007). Studies were further undertaken and Bacillus coagulans CCI was isolated as autochthonous probiotic bacteria from the fish gut of Catla catla (Bhatnagar, Raparia, and Kumari, 2012) and evaluated that the effect of dietary incorporation of isolated Bacillus coagulans CC1 resulted in enhanced growth performance and nutrient retention when incorporated in fish diet at an optimum level (Bhatnagar and Raparia, 2014). C. catla is one of the important Indian Major Carps and a very constitutive part of our food chain. Its economic importance is gradually declining due to its disease outbreak. Plant extracts along with probiotics can strongly make an alternative way to replace synthetic immune stimulants and enhance the aquatic health leading to sustainable aquaculture. However, to the best of author's knowledge, literature is not available demonstrating the use of plant extract and autochthonous probiotics in synergism. Therefore, the current study was undertaken to assess the impact of $M$. piperita and B. coagulans on growth performance, nutritional physiology, and immunity of C. Catla.

\section{Materials and methods}

\section{Sample collection}

The experimental fish fingerlings of Catla catla were procured from the fish farm in the vicinity of Kurukshetra
(Sultan Fish Farm, Village Butana, Nilokheri, Kurukshetra, India) and were Acclimated for 15 days. Two experiments (Table 1) were conducted to evaluate the effect of medicinal plant Mentha piperita (Peppermint) with or without probiotic bacterium Bacillus coagulans on growth performance and associated nutritional physiology of Catla catla in the Aquaculture Research Unit, Department of Zoology, Kurukshetra University, Kurukshetra.

\section{Feed preparation}

Peppermint leaves (as leaves contain its active biological components) were collected from its natural habitat and identified properly. Leaves were dried for three to four days initially in the shade and then in an oven for $55^{\circ} \mathrm{C}$; thereafter, they were crushed into powder form using a pestle and mortar. Uniform powdered form of peppermint leaves was obtained by using a fine-meshed sieve and thoroughly mixed directly with basal diet. Basal diet was prepared by using groundnut oil cake $(650 \mathrm{~g})$, rice bran (32 g), duckweed (266 g), wheat flour (32 g), and mineral mixture $(10 \mathrm{~g})$. For Experiment 1 , five isonitrogenous and isocaloric diets (C1, P1, P2, P3, P4) of 40\% protein content were prepared (Table 2). Basal diet served as control. In Diet P1 to P4, Peppermint was added at $2 \mathrm{~g} \mathrm{Kg}^{-1}, 4 \mathrm{~g} \mathrm{Kg}^{-1}, 6 \mathrm{~g} \mathrm{Kg}^{-1}$, and $8 \mathrm{~g} \mathrm{Kg}^{-1}$ of feed, respectively. For Experiment 2, five isonitrogenous and isocaloric diets (C2, P1, PP2, PP3, PP4) of 40\% protein content were prepared (Table 2). Control (C2) diet was prepared by adding probiotics Bacillus coagulans at the rate of $3000 \mathrm{CFU} \mathrm{g}^{-1}$ of feed to basal diet. In Diet PP1 to PP4, incorporation of probiotics Bacillus coagulans at the rate of $3000 \mathrm{CFU} \mathrm{g}{ }^{-1}$ of feed along with peppermint was done at $2 \mathrm{~g} \mathrm{Kg}^{-1}, 4 \mathrm{~g} \mathrm{Kg}^{-1}, 6 \mathrm{~g} \mathrm{Kg}^{-1}$, and $8 \mathrm{~g} \mathrm{Kg}^{-1}$ of feed, respectively. All ingredients were thoroughly grounded, sieved, mixed, and then followed by preparation of dough homogeneously. Feed were prepared in the form of pellets by using a pelletizer. Feed was then oven-dried at the temperature of $60^{\circ} \mathrm{C}$ and stored in air tight containers. Probiotics-supplemented diet was stored at $4{ }^{\circ} \mathrm{C}$ to maintain it contamination-free.

\section{Experimental setup}

The experiment was conducted in the Aquaculture Research Unit, Department of Zoology, Kurukshetra

Table 1 Experimental details

\begin{tabular}{|c|c|c|c|c|}
\hline \multicolumn{3}{|c|}{ Experiment 1} & \multicolumn{2}{|c|}{ Experiment 2} \\
\hline 1 & Diet C1 & No M. piperita, No probiotic bacterium B. coagulans & Diet C2 & No M. piperita but probiotic bacterium B. coagulans @ 3000 CFU g ${ }^{-1}$ \\
\hline 2 & Diet P1 & M. piperita @ $2 \mathrm{~g} \mathrm{Kg}^{-1}$ & Diet PP1 & M. piperita@ $2 \mathrm{~g} \mathrm{Kg}^{-1}+$ probiotic bacterium B. coagulans @ $3000 \mathrm{CFU} \mathrm{g}^{-1}$ \\
\hline 3 & Diet P2 & M. piperita@ @ $\mathrm{g} \mathrm{Kg}^{-1}$ & Diet PP2 & M. piperita@4 $\mathrm{g} \mathrm{Kg}^{-1}+$ probiotic bacterium B. coagulans @ 3000 CFU g \\
\hline 4 & Diet P3 & M. piperita @ $6 \mathrm{~g} \mathrm{Kg}^{-1}$ & Diet PP3 & M. piperita@6 g kg + probiotic bacterium B. coagulans @ 3000 CFU g \\
\hline 5 & Diet P4 & M. piperita @ $8 \mathrm{~g} \mathrm{Kg}^{-1}$ & Diet PP4 & M. piperita@8 $\mathrm{g} \mathrm{Kg}^{-1}$ + probiotic bacterium B.coagulans @ 3000 CFU g ${ }^{-1}$ \\
\hline
\end{tabular}


Table 2 Proximate composition (\% dry weight basis) of experimental diets containing Mentha piperita without or with probiotic bacterium Bacillus coagulans (Experiment 1 and Experiment 2)

\begin{tabular}{|c|c|c|c|c|c|}
\hline \multirow{2}{*}{$\begin{array}{l}\text { Proximate analysis } \\
\text { Experiment } 1\end{array}$} & \multicolumn{5}{|c|}{ Dietary treatments } \\
\hline & & & & & \\
\hline & $\mathrm{C} 1$ & P1 & P2 & P3 & P4 \\
\hline Crude protein (\%) & $40.97 \pm 0.030^{A}$ & $41.27 \pm 0.040^{A}$ & $41.47 \pm 0.030^{\mathrm{A}}$ & $41.88 \pm 0.040^{\mathrm{A}}$ & $41.20 \pm 0.032^{\mathrm{A}}$ \\
\hline Crude fat (\%) & $9.05 \pm 0.030^{B}$ & $9.11 \pm 0.040^{B}$ & $9.28 \pm 0.050^{A}$ & $9.29 \pm 0.020^{A}$ & $9.30 \pm 0.030^{A}$ \\
\hline Crude fiber (\%) & $6.28 \pm 0.030^{c}$ & $6.43 \pm 0.040^{A B}$ & $6.38 \pm 0.020^{B C}$ & $6.44 \pm 0.020^{A B}$ & $6.52 \pm 0.020^{A}$ \\
\hline Total ash (\%) & $7.34 \pm 0.030^{A}$ & $7.19 \pm 0.050^{B}$ & $7.29 \pm 0.020^{\mathrm{AB}}$ & $7.22 \pm 0.020^{\mathrm{AB}}$ & $7.28 \pm 0.020^{\mathrm{AB}}$ \\
\hline Moisture (\%) & $4.47 \pm 0.060^{A}$ & $4.29 \pm 0.010^{B C}$ & $4.20 \pm 0.030^{C}$ & $4.24 \pm 0.030^{B C}$ & $4.35 \pm 0.020^{A B}$ \\
\hline Nitrogen free extract (\%) & $39.23 \pm 0.020^{A}$ & $38.89 \pm 0.080^{B}$ & $38.67 \pm 0.070^{B}$ & $38.14 \pm 0.080^{c}$ & $38.62 \pm 0.030^{B}$ \\
\hline Gross energy $\left(\mathrm{kJ} \mathrm{g}^{-1}\right)$ & $19.99 \pm 0.010^{C}$ & $20.03 \pm 0.010^{C}$ & $20.10 \pm 0.010^{\mathrm{AB}}$ & $20.11 \pm 0.010^{A}$ & $20.04 \pm 0.010^{B C}$ \\
\hline \multicolumn{6}{|l|}{ Experiment 2} \\
\hline & $\mathrm{C} 2$ & PP1 & PP2 & PP3 & PP4 \\
\hline Crude Protein (\%) & $40.97 \pm 0.030^{A}$ & $41.27 \pm 0.040^{A}$ & $41.47 \pm 0.030^{A}$ & $41.88 \pm 0.040^{\mathrm{A}}$ & $41.20 \pm 0.032^{\mathrm{A}}$ \\
\hline Crude fat (\%) & $9.21 \pm 0.010^{C}$ & $9.31 \pm 0.020^{B}$ & $9.42 \pm 0.010^{A}$ & $9.47 \pm 0.010^{A}$ & $9.41 \pm 0.010^{A}$ \\
\hline Crude fiber (\%) & $6.31 \pm 0.010^{c}$ & $6.37 \pm 0.010^{B C}$ & $6.44 \pm 0.010^{B}$ & $6.54 \pm 0.020^{A}$ & $6.56 \pm 0.010^{A}$ \\
\hline Total Ash (\%) & $7.32 \pm 0.020^{\mathrm{AB}}$ & $7.25 \pm 0.030^{B}$ & $7.35 \pm 0.020^{\mathrm{AB}}$ & $7.38 \pm 0.030^{A}$ & $7.34 \pm 0.010^{A B}$ \\
\hline Moisture (\%) & $4.30 \pm 0.020^{B}$ & $4.34 \pm 0.020^{\mathrm{AB}}$ & $4.43 \pm 0.010^{A}$ & $4.45 \pm 0.040^{\mathrm{A}}$ & $4.33 \pm 0.020^{\mathrm{AB}}$ \\
\hline Nitrogen free extract (\%) & $39.20 \pm 0.070^{A}$ & $38.71 \pm 0.060^{B}$ & $38.23 \pm 0.030^{c}$ & $37.66 \pm 0.070^{D}$ & $38.48 \pm 0.040^{B C}$ \\
\hline Gross energy $\left(\mathrm{kJ} \mathrm{g}^{-1}\right)$ & $20.05 \pm 0.010^{B}$ & $20.07 \pm 0.003^{A B}$ & $20.08 \pm 0.010^{A B}$ & $20.10 \pm 0.010^{A}$ & $20.06 \pm 0.010^{A B}$ \\
\hline
\end{tabular}

Note: All values are mean \pm S.E. of mean. Values with different superscript in the same row are significantly $(P<0.05)$ different $(T u k e y ' s$ honest test)

University, Kurukshetra. The Acclimated fingerlings were distributed in different aquaria $(60 \times 30 \times 30 \mathrm{~cm}) /$ plastic tubs (50 L capacity) containing 10 fingerlings each under laboratory conditions $\left(24 \pm 1^{\circ} \mathrm{C}\right)$. All groups of fishes in both experiments were fed daily at $3 \%$ BW in 2 installments at 10:00 and 17:00 h for 90 days provided 50-60\% exchange of dechlorinated water daily along with constant aeration. Uneaten food was collected after $4 \mathrm{~h}$ of feeding for further analysis. Every individual fingerling was weighed at the start of the experiment, after every 15 days, and at the end of the experiment for the assessment of growth performance parameters.

\section{Apparent protein digestibility (APD) and proximate analysis}

Fecal matter was also collected daily and then ovendried for determination of apparent protein digestibility with the help of chromic oxide $\left(\mathrm{Cr}_{2} \mathrm{O}_{3}\right)$ as a digestibility marker according to $\mathrm{Cho}$, Slinger, and Bayley (1982). Proximate analysis was performed following AOAC (1995). In proximate analysis, moisture content (evaporation in hot air oven at $95^{\circ} \mathrm{C}$ for 48 h), crude protein (by Kjeldahl method), crude fat (by using petroleum ether in Soxhlet apparatus), and ash content (by incineration in muffle furnace at $550{ }^{\circ} \mathrm{C}$ for $4 \mathrm{~h}$ ) were analyzed for both carcass and for feed. Crude fiber (by acid/alkali digestion method) was also analyzed for fish feed. NFE was estimated by subtraction method; NFE $=100-($ crude protein + fat + moisture).

\section{Water quality}

During experimentation, at every 15th day, water quality parameters likewise temperature, $\mathrm{pH}$, dissolved oxygen (DO), chloride, calcium, total alkalinity, electrical conductivity, and total hardness were analyzed following APHA (1998) to check the effect of feed on water characteristics.

\section{Ammonia and phosphate excretion in holding water}

At the end of 90 days feeding trials, fishes were offered the same diet and were allowed for $2 \mathrm{~h}$ to consume the feed. After $2 \mathrm{~h}$, excess of feed was removed and fixed levels of water of all aquaria were maintained for experiments. After that, water samples from each aquarium were collected at 2-h intervals to evaluate the excretory levels of total ammonia (N$\left.\mathrm{NH}_{4}\right)$ and reactive orthophosphate $\left(\mathrm{o}-\mathrm{PO}_{4}\right)$ following APHA (1998). The quantity of nitrogen and phosphate excreted by fish in holding water were calculated as follows: 


$$
\begin{aligned}
& \text { Total } \mathrm{N}-\mathrm{NH}_{4} / \mathrm{o}-\mathrm{PO}_{4} \text { excretion } \\
& \left(\mathrm{mg} \mathrm{kg}^{-1} \mathrm{BW} 2 \mathrm{~h}^{-1}\right) \\
& =\frac{\left[\left(\mathrm{N}^{-} \mathrm{NH}_{4} / \mathrm{o}-\mathrm{PO}_{4}\right)_{120}-\left(\mathrm{N}-\mathrm{NH}_{4} / \mathrm{o}-\mathrm{PO}_{4}\right)_{0}\right] \times a}{\text { Fish biomass } / \mathrm{kg}}
\end{aligned}
$$

where $\left(\mathrm{N}-\mathrm{NH}_{4} / \mathrm{o}-\mathrm{PO}_{4}\right)_{0}$ and $\left(\mathrm{N}-\mathrm{NH}_{4} / \mathrm{o}-\mathrm{PO}_{4}\right)_{120}=$ Concentration at times 0 and $120 \mathrm{~min}(2 \mathrm{~h})$ post feeding.

$a=$ amount of holding water $(\mathrm{L})$ in which fishes were kept.

\section{Growth parameters}

Various growth parameters were calculated for evaluating dietary performances and nutritional indices (Garg, Bhatnagar, Kalla, and Johal, 2002).

\section{Intestinal enzyme activities}

At the end of the feeding trial, two fingerlings from each treatment were taken, kept on ice tray, and their intestines were removed which was further processed for the determination of different intestinal enzyme activities such as protease (Walter, 1984), cellulase (Sadasivam and Manickam, 1996), and amylase (Sawhney and Singh, 2000).

\section{Collection of blood}

Blood sample was collected from 5-6 fish from each treatment (fish were anesthetized using MS 222, SigmaAldrich) so as to pool the blood for the immunological, serological, and hematological diagnosis by caudal vein/ heart puncture using a heparinized syringe, which was previously rinsed with EDTA solution and transferred immediately to an Eppendorf tube.

\section{Hematological diagnosis}

The collected blood samples were used for the estimation of total leucocyte count (TLC) and total erythrocyte count (TEC) with the help of hemocytometer using a Neubauer's counting chamber following Dacie and Lewis (1963).

\section{Phagocytic assay}

The phagocytic assay was performed according to the method of Siwicki, Anderson, and Rumsey (1994) and Park and Jeong (1996) with a slight modification. Blood samples were collected for determination of the phagocytic cells and phagocytosed bacteria for each treatment. Phagocytic activity (PA) and phagocytic index (PI) was determined by enumerating 100 phagocytes per slide under a microscope, and the average of three slides was calculated:

Phagocytic activity (i.e., percentage of cell with engulfed bacteria) $=$ Number of phagocytic cells with engulfed bacteria/Number of phagocytic cells $\times 100$.

Phagocytic index (i.e., number of engulfed bacteria per cell $)=$ Number of engulfed bacteria/Number of phagocytic cells.

\section{Nitroblue tetrazolium (NBT) assay}

The oxygen radical production by blood phagocytes during respiratory burst activity was measured through Nitroblue tetrazolium (NBT) assay using spectrophotometer at $540 \mathrm{~nm}$ as described by Anderson and Siwicki (1995).

\section{Serum bactericidal activity}

Serum from blood samples of each treatment was collected for serum bactericidal activity (Kajita, Sakai, Atsuta, and Kobayashi, 1990). The number of viable bacteria was calculated by counting the colonies from the resultant incubated mixture on TSA plates in duplicate (two plates per sample) after 24-h incubation. Less bacterial count will show better serum bactericidal activity.

\section{Total serum protein determination}

The serum protein was determined according to Gornall, Bardawill, and David (1949). The blood samples were taken in a test tube which was not heparinized and allowed to stay until the clotted blood settled down. The serum was then transferred into another test tube. The serum was centrifuged for $10 \mathrm{~min}$ at $3000 \mathrm{rpm}$. After that, $0.1 \mathrm{ml}$ of serum was treated with $5 \mathrm{ml}$ of Gornal reagent (1.5 g CuSO $4.5 \mathrm{H}_{2} \mathrm{O}, 6 \mathrm{~g} \mathrm{KNaC}_{4} \mathrm{H}_{4} \mathrm{O}_{6}, 500 \mathrm{ml} \mathrm{H} \mathrm{H}_{2} \mathrm{O}, 300 \mathrm{ml}$ of $10 \% \mathrm{NaOH}$ ) and was shaken for $1 \mathrm{~min}$. The absorbance of the mixture was determined spectrophotometrically at $540 \mathrm{~nm}$, and the serum protein values were deduced by using a standard curve.

\section{Challenge trial}

From each treatment, 10 fishes were challenged with $A$. hydrophila after feeding trial of 90 days (Austin, Stuckey, Robertson, Effendi, and Griffith, 1995). Percent survival was measured for 10 days based on the observation that mortality reached its elevation after 1 week (Sahoo, De, Ghosh, and Maitra, 1998) and relative percentage survival (RPS) was calculated by the following formula (Ellis, 1988)

$$
\begin{aligned}
& \text { Relative Percentage Survival } \\
& \quad=1-\frac{\text { Percent mortality in treated group }}{\text { Percent mortality in control group }} \times 100
\end{aligned}
$$

Kaplan-Meier survivorship curve was also constructed on MS Excel to show the pattern of fingerlings fed on different supplemented diets of $M$. piperita with and without probiotic in both experiments during challenge trial with the fish pathogenic bacterium, A. hydrophila, for ten days (Kaplan and Meier, 1958).

\section{Statistical analysis}

Data were subjected to one way ANOVA followed by Tukey's honest test to test the significant differences between different dietary treatments (Tukey, 1977). Statistical significance was settled at a probability 
value of $P<0.05$. All statistics were performed using SPSS Version 18.0.

\section{Results \\ Growth and digestibility \\ Experiment 1}

The growth parameters such as weight gain (WG), specific growth rate (SGR), protein efficiency ratio (PER), gross conversion efficiency (GCE), feed conversion ratio (FCR), and apparent protein digestibility (APD) of fish fed with a distinctive level of peppermint-supplemented diets are shown in Table 3. ANOVA followed by Tukey's honest test revealed that WG, SGR, PER, GCE, and APD values were significantly $(P<0.05)$ high in all treatments in comparison to control with the highest value in dietary treatment P3. While FCR values were significantly low in all the treatments in comparison to control.

\section{Experiment 2}

The WG, SGR, PER, GCE, FCR, and APD of fish fed with different inclusion levels of peppermint-supplemented diets with probiotic bacterium Bacillus coagulans are shown in Table 4. Significant increase in the values of these parameters was realized in treatment PP3 for this experiment. Significantly, low FCR values were recognized in fishes fed on a diet containing $6 \mathrm{~g} \mathrm{~kg}^{-1}$ of $M$. piperita along with probiotic bacterium Bacillus coagulans at the rate of (@) $3000 \mathrm{CFU} \mathrm{g}{ }^{-1}$ in comparison to other treatments and control.

\section{Intestinal enzyme activities \\ Experiment 1}

Specific protease, amylase, and cellulase activities were significantly high in those fingerlings whose diet was supplemented with $M$. piperita in comparison to control with highest value in dietary treatment P3.

\section{Experiment 2}

After completion of the experiment, the increase of intestinal enzymes activity of fishes fed on different peppermint-supplemented diets along with probiotic bacterium Bacillus coagulans as comparison to control diet (Table 4$)$ was clearly examined. Significantly $(P<$ 0.05 ) higher intestinal enzymes activity was revealed in treatment PP3 in this experiment.

\section{Water quality characteristics \\ Experiment 1}

No significant variations were observed in water quality parameters. These parameters do not show any definitive trend with varying inclusion of peppermint in supplemented diet (Table 5). However, significantly $(\mathrm{P}<0.05)$ low values of total ammonia excretion $(454.33 \pm 2.18)$ and reactive orthophosphate production $(290.67 \pm 1.20)$ were recognized in fish fed on

Table 3 Effect of dietary inclusion of M. piperita without probiotic bacterium B. coagulans on growth performance and intestinal enzyme activities of C. catla (Experiment 1)

\begin{tabular}{|c|c|c|c|c|c|}
\hline \multirow[t]{2}{*}{ Growth parameters } & \multicolumn{5}{|c|}{ Dietary treatments } \\
\hline & $\mathrm{C1}$ & P1 & $\mathrm{P} 2$ & P3 & P4 \\
\hline Initial weight (g) & $1.04 \pm 0.01$ & $1.01 \pm 0.004$ & $1.02 \pm 0.003$ & $1.05 \pm 0.020$ & $1.06 \pm 0.002$ \\
\hline Final weight (g) & $2.50 \pm 0.010^{c}$ & $2.79 \pm 0.360^{B}$ & $2.90 \pm 0.090^{\mathrm{AB}}$ & $3.05 \pm 0.050^{A}$ & $2.78 \pm 0.250^{B}$ \\
\hline Weight gain (g) & $1.47 \pm 0.020^{C}$ & $1.78 \pm 0.030^{\mathrm{AB}}$ & $1.87 \pm 0.080^{\mathrm{AB}}$ & $2.00 \pm 0.070^{\mathrm{A}}$ & $1.72 \pm 0.030^{B}$ \\
\hline Growth \% gain in BW & $\begin{array}{l}141.09 \pm \\
4.150^{C}\end{array}$ & $\begin{array}{l}175.82 \pm \\
3.170^{\mathrm{AB}}\end{array}$ & $\begin{array}{l}181.92 \pm \\
7.590^{\mathrm{AB}}\end{array}$ & $\begin{array}{l}192.18 \pm \\
9.920^{A}\end{array}$ & $\begin{array}{l}161.48 \pm \\
2.240^{B C}\end{array}$ \\
\hline Growth per day in BW & $0.92 \pm 0.030^{C}$ & $1.04 \pm 0.010^{\mathrm{AB}}$ & $1.05 \pm 0.040^{\mathrm{AB}}$ & $1.09 \pm 0.050^{\mathrm{A}}$ & $0.99 \pm 0.010^{B}$ \\
\hline Specific growth rate (SGR) $\left(\% \mathrm{BW} \mathrm{d}^{-1}\right)$ & $0.38 \pm 0.010^{C}$ & $0.44 \pm 0.010^{\mathrm{AB}}$ & $0.45 \pm 0.010^{\mathrm{AB}}$ & $0.46 \pm 0.150^{A}$ & $0.42 \pm 0.003^{\mathrm{BC}}$ \\
\hline Feed conversion ratio (FCR) & $3.13 \pm 0.090^{\mathrm{A}}$ & $2.74 \pm 0.030^{B}$ & $2.68 \pm 0.110^{B}$ & $2.58 \pm 0.080^{B}$ & $2.81 \pm 0.050^{\mathrm{AB}}$ \\
\hline Gross conversion efficiency (GCE) & $0.32 \pm 0.010^{C}$ & $0.36 \pm 0.004^{\mathrm{AB}}$ & $0.37 \pm 0.020^{\mathrm{AB}}$ & $0.39 \pm 0.010^{\mathrm{A}}$ & $0.36 \pm 0.010^{B}$ \\
\hline Protein efficiency ratio (PER) & $0.04 \pm 0.001^{C}$ & $0.04 \pm 0.001^{\mathrm{AB}}$ & $0.05 \pm 0.002^{\mathrm{AB}}$ & $0.05 \pm 0.002^{\mathrm{A}}$ & $0.04 \pm 0.003^{B C}$ \\
\hline Apparent protein digestibility (APD) (\%) & $74.25 \pm 0.620^{B}$ & $74.32 \pm 0.600^{\mathrm{B}}$ & $75.68 \pm 0.530^{\mathrm{AB}}$ & $77.67 \pm 0.420^{\mathrm{A}}$ & $75.50 \pm 0.670^{\mathrm{AB}}$ \\
\hline Protease activity ( $\mathrm{mg}$ of tyrosine liberated $\mathrm{mg}$ of protein $^{-1} \mathrm{~h}^{-1}$ ) & $1.77 \pm 0.010^{\mathrm{D}}$ & $2.14 \pm 0.010^{C}$ & $2.21 \pm 0.010^{B}$ & $2.33 \pm 0.010^{\mathrm{A}}$ & $2.14 \pm 0.010^{c}$ \\
\hline Amylase activity (mg of maltose liberated mgof protein ${ }^{-1} \mathrm{~h}^{-1}$ ) & $1.29 \pm 0.020^{\mathrm{E}}$ & $1.47 \pm 0.010^{\mathrm{D}}$ & $1.72 \pm 0.010^{B}$ & $2.09 \pm 0.010^{\mathrm{A}}$ & $1.57 \pm 0.010^{C}$ \\
\hline $\begin{array}{l}\text { Cellulase activity (mg of glucose liberated } m g \text { of protein }{ }^{-1} \\
h^{-1} \text { ) }\end{array}$ & $0.97 \pm 0.010^{E}$ & $1.11 \pm 0.010^{\mathrm{D}}$ & $1.20 \pm 0.010^{C}$ & $1.40 \pm 0.010^{\mathrm{A}}$ & $1.29 \pm 0.010^{B}$ \\
\hline
\end{tabular}

Weight gain $(g)=W_{2}-W_{1}$; Growth \% gain in $B W=\left(W_{2}-W_{1}\right) / W_{1} \times 100 ;$ Growth per day in $\% B W=\left\{2\left(W_{2}-W_{1}\right) / t\left(W_{1}+W_{2}\right)\right\} \times 100 ; S G R=\left(\ln W_{2}-\ln W_{1}\right) / t \times 100$; $\mathrm{FCR}=$ Feed Offered (Dry wt.) (g)/Body weight gain (Wet wt.) (g); GCE = Body weight gain (Wet wt.) (g)/Feed Offered (Dry wt.) (g); PER=Wet Weight Gain (g)/Crude protein fed (\%)

Where, $\mathrm{W}_{1}=$ Initial Weight $(\mathrm{g}), \mathrm{W}_{2}=$ Final Weight $(\mathrm{g})$, Duration of experiment (No. of days)

Note: All values are mean \pm S.E. of mean. Values with different superscript in the same row are significantly $(P<0.05)$ different (Tukey's honest test) 
Table 4 Effect of dietary inclusion of M. piperita with probiotic bacterium B. coagulans on growth performance and intestinal enzyme activities of Catla catla (Experiment 2)

\begin{tabular}{|c|c|c|c|c|c|}
\hline \multirow[t]{2}{*}{ Growth rarameters } & \multicolumn{5}{|c|}{ Dietary treatments } \\
\hline & $\mathrm{C} 2$ & PP1 & PP2 & PP3 & PP4 \\
\hline Initial weight (g) & $1.05 \pm 0.010$ & $1.03 \pm 0.010$ & $1.05 \pm 0.010$ & $1.06 \pm 0.002$ & $1.03 \pm 0.010$ \\
\hline Final weight (g) & $2.81 \pm 0.010^{C}$ & $2.93 \pm 0.050^{B C}$ & $3.02 \pm 0.060^{\mathrm{AB}}$ & $3.21 \pm 0.150^{A}$ & $2.78 \pm 0.390^{C}$ \\
\hline Weight gain (g) & $1.75 \pm 0.001^{C}$ & $1.90 \pm 0.040^{B C}$ & $1.97 \pm 0.080^{\mathrm{AB}}$ & $2.15 \pm 0.010^{A}$ & $1.75 \pm 0.040^{C}$ \\
\hline Growth \% gain in BW & $166.53 \pm 2.640^{B}$ & $184.27 \pm 1.940^{\mathrm{AB}}$ & $188.10 \pm 9.410^{A B}$ & $202.58 \pm 1.340^{\mathrm{A}}$ & $169.73 \pm 3.220^{B}$ \\
\hline Growth per day in BW & $1.01 \pm 0.010^{B}$ & $1.07 \pm 0.010^{\mathrm{AB}}$ & $1.07 \pm 0.030^{\mathrm{AB}}$ & $1.12 \pm 0.003^{\mathrm{A}}$ & $1.02 \pm 0.010^{B}$ \\
\hline Specific growth rate (SGR) (\% BW d ${ }^{-1}$ ) & $0.42 \pm 0.003^{B}$ & $0.45 \pm 0.003^{\mathrm{AB}}$ & $0.46 \pm 0.020^{\mathrm{AB}}$ & $0.48 \pm 0.003^{\mathrm{A}}$ & $0.43 \pm 0.010^{B}$ \\
\hline Feed conversion ratio (FCR) & $2.76 \pm 0.030^{\mathrm{AB}}$ & $2.64 \pm 0.030^{C}$ & $2.74 \pm 0.090^{\mathrm{AB}}$ & $2.53 \pm 0.020^{B C}$ & $2.84 \pm 0.010^{\mathrm{A}}$ \\
\hline Gross conversion efficiency (GCE) & $0.36 \pm 0.004^{B}$ & $0.38 \pm 0.004^{\mathrm{AB}}$ & $0.37 \pm 0.010^{B}$ & $0.40 \pm 0.003^{\mathrm{A}}$ & $0.35 \pm 0.002^{B}$ \\
\hline Protein efficiency ratio (PER) & $0.04 \pm 0.003^{C}$ & $0.05 \pm 0.001^{B C}$ & $0.05 \pm 0.002^{\mathrm{AB}}$ & $0.05 \pm 0.0003^{\mathrm{A}}$ & $0.04 \pm 0.001^{c}$ \\
\hline Apparent protein digestibility (APD) (\%) & $74.64 \pm 0.490^{B}$ & $77.43 \pm 0.640^{\mathrm{AB}}$ & $78.37 \pm 0.630^{A}$ & $78.96 \pm 0.680^{A}$ & $76.62 \pm 0.570^{\mathrm{AB}}$ \\
\hline Protease activity ( $\mathrm{mg}$ of tyrosine liberated $\mathrm{mg}$ of protei $^{-1} \mathrm{~h}^{-1}$ ) & $2.23 \pm 0.020^{c}$ & $2.30 \pm 0.020^{B}$ & $2.33 \pm 0.020^{B}$ & $2.62 \pm 0.020^{\mathrm{A}}$ & $2.27 \pm 0.020^{B C}$ \\
\hline Amylase activity (mg of maltose liberated $\mathrm{mg}$ of protein ${ }^{-1} \mathrm{~h}^{-1}$ ) & $1.68 \pm 0.020^{D}$ & $1.84 \pm 0.010^{\mathrm{E}}$ & $2.13 \pm 0.020^{B}$ & $2.40 \pm 0.010^{\mathrm{A}}$ & $1.93 \pm 0.010^{C}$ \\
\hline Cellulase activity ( $\mathrm{mg}$ of glucose liberated $\mathrm{mg}$ of protein ${ }^{-1} \mathrm{~h}^{-1}$ ) & $1.20 \pm 0.020^{\mathrm{E}}$ & $1.35 \pm 0.010^{\mathrm{D}}$ & $1.47 \pm 0.020^{C}$ & $1.73 \pm 0.010^{\mathrm{A}}$ & $1.55 \pm 0.010^{B}$ \\
\hline
\end{tabular}

Weight gain $(\mathrm{g})=\mathrm{W}_{2}-\mathrm{W}_{1}$; Growth \% gain in $\mathrm{BW}=\left(\mathrm{W}_{2}-\mathrm{W}_{1}\right) / \mathrm{W}_{1} \times 100$; Growth per day in $\% \mathrm{BW}=\left\{2\left(\mathrm{~W}_{2}-\mathrm{W}_{1}\right) / t\left(\mathrm{~W}_{1}+\mathrm{W}_{2}\right)\right\} \times 100 ; \mathrm{SGR}=\left(\ln \mathrm{W}_{2}-\ln \mathrm{W}_{1}\right) / t \times 100$; FCR = Feed Offered (Dry wt.) (g)/Body weight gain (Wet wt.) (g); GCE = Body weight gain (Wet wt.) (g)/Feed Offered (Dry wt.) (g); PER =Wet Weight Gain (g)/Crude protein fed (\%)

Where, $\mathrm{W}_{1}=$ Initial Weight $(\mathrm{g}), \mathrm{W}_{2}=$ Final Weight $(\mathrm{g})$, Duration of experiment (No. of days)

Note: All values are mean \pm S.E. of mean. Values with different superscript in the same row are significantly $(P<0.05)$ different (Tukey's honest test)

peppermint-supplemented diet @ $6 \mathrm{~g} \mathrm{Kg}^{-1}$ (treatment P3) compared to all other treatments in the first experiment.

\section{Experiment 2}

Water quality parameters showed insignificant variations among all treatments. These parameters do not follow any specific trend with different diets containing different inclusion level of peppermint with probiotic bacterium Bacillus coagulans (Table 5). Despite this, PP3-treated fingerlings showed significantly $(P<$ $0.05)$ low values of ammonia excretion $(363.33 \pm 2.03)$ and orthophosphate production $(206.67 \pm 1.76)$ than all other treatments.

\section{Whole body proximate analysis \\ Experiment 1}

After 90 days of feeding trial, final carcass composition of C. catla fingerlings of all treatments were analyzed and presented in Table 6. In this experiment, the carcass composition of test animals containing peppermint showed a significant increase in carcass protein, carcass lipid, and total gross energy in comparison to control fingerlings. Treatment P3-fed group fingerlings showed a significant increase in carcass protein $(15.93 \pm 0.03)$. Lipid content $(6.99 \pm 0.03)$ and gross energy $(8.79 \pm 0.01)$ were found to be highest in fishes of treatment P3.

\section{Experiment 2}

In this experiment, the carcass composition of fingerlings fed on different incorporation levels of peppermint with probiotic bacterium Bacillus coagulans was analyzed and given in Table 6. Significantly highest carcass protein $(16.14 \pm 0.04)$ was observed in fingerlings of PP3 followed by PP2 (15.91 \pm 0.02$)$-fed group fingerlings in the second experiment. Lipid values $(7.00 \pm 0.03)$ were significantly high in treatment PP3 followed by treatment PP4 $(6.95 \pm 0.02)$ and gross energy was also observed significantly higher in treatment PP3 followed by treatment PP2 fed group fingerlings.

\section{Hematological parameters Experiment 1}

The total erythrocytes were significantly $(P<0.05)$ higher in fishes fed on diet P3 (Fig. 1a). The total leucocytes were also significantly $(P<0.05)$ high in fishes fed on diet P3 $(42.67 \pm 0.88)$ than other treatments and control (Fig. 1b).

\section{Experiment 2}

In this experiment, the values of total erythrocytes and leucocytes of fingerlings fed on different diets containing different inclusion level of peppermint along with probiotic bacterium are shown in Fig. 1a, b. The total erythrocytes $(2.06 \pm 0.02)$ and total leucocytes count $(46.33 \pm 1.45)$ of treatment PP3-fed group 
Table 5 Effect of fish fed on M. piperita-supplemented diet without or with probiotic bacterium B. coagulans on water quality characteristics (Experiment 1 and Experiment 2)

\begin{tabular}{|c|c|c|c|c|c|}
\hline \multirow{2}{*}{$\frac{\text { Physico-chemical parameters }}{\text { Experiment } 1}$} & \multicolumn{5}{|l|}{ Dietary treatments } \\
\hline & & & & & \\
\hline & $\mathrm{C} 1$ & P1 & P2 & P3 & P4 \\
\hline Dissolved oxygen (DO) $\mathrm{mg} \mathrm{L}^{-1}$ & $6.71 \pm 0.040^{A}$ & $6.73 \pm 0.130^{A}$ & $6.71 \pm 0.060^{A}$ & $6.45 \pm 0.020^{\mathrm{A}}$ & $6.60 \pm 0.040^{A}$ \\
\hline $\mathrm{pH}$ & $7.60 \pm 0.040^{A}$ & $7.57 \pm 0.130^{A}$ & $7.59 \pm 0.060^{\mathrm{A}}$ & $7.66 \pm 0.020^{A}$ & $7.42 \pm 0.050^{A}$ \\
\hline Temperature $\left({ }^{\circ} \mathrm{C}\right)$ & $26.50 \pm 0.200^{A}$ & $26.23 \pm 0.080^{A}$ & $26.25 \pm 0.070^{A}$ & $26.33 \pm 0.220^{A}$ & $26.13 \pm 0.050^{\mathrm{A}}$ \\
\hline Conductivity ( $\mu$ mhos $\mathrm{cm}^{-1}$ ) & $662.22 \pm 0.710^{A}$ & $664.17 \pm 5.170^{A}$ & $668.00 \pm 2.080^{A}$ & $660.56 \pm 12.650^{A}$ & $661.50 \pm 3.770^{A}$ \\
\hline Alkalinity $\left(\mathrm{mgL}^{-1}\right)$ & $191.11 \pm 1.440^{\mathrm{A}}$ & $188.67 \pm 2.400^{\mathrm{A}}$ & $187.00 \pm 2.500^{\mathrm{A}}$ & $186.22 \pm 1.730^{\mathrm{A}}$ & $190.33 \pm 2.510^{\mathrm{A}}$ \\
\hline Chloride $\left(\mathrm{mg} \mathrm{L}^{-1}\right)$ & $39.59 \pm 0.500^{A}$ & $38.43 \pm 0.430^{A}$ & $38.22 \pm 0.330^{A}$ & $38.16 \pm 0.140^{A}$ & $39.37 \pm 0.240^{A}$ \\
\hline Calcium $\left(\mathrm{mgL}^{-1}\right)$ & $18.73 \pm 0.680^{A}$ & $18.33 \pm 0.170^{A}$ & $19.39 \pm 0.490^{A}$ & $19.11 \pm 0.280^{A}$ & $18.78 \pm 0.480^{A}$ \\
\hline Hardness $\left(\mathrm{mgL}^{-1}\right)$ & $83.22 \pm 0.730^{A}$ & $80.11 \pm 0.950^{A}$ & $79.78 \pm 0.950^{A}$ & $78.33 \pm 1.680^{A}$ & $78.00 \pm 1.900^{\mathrm{A}}$ \\
\hline Total $\mathrm{NH}_{3}-\mathrm{N}$ excretion ( $\mathrm{mg} \mathrm{Kg}^{-1} \mathrm{BW}$ day ${ }^{-1}$ ) & $766.67 \pm 2.330^{\mathrm{A}}$ & $521.67 \pm 2.030^{c}$ & $459.67 \pm 3.050^{\mathrm{D}}$ & $454.33 \pm 2.180^{\mathrm{E}}$ & $568.67 \pm 3.180^{B}$ \\
\hline Total o- $\mathrm{PO}_{4}$ production $\left(\mathrm{mg} \mathrm{Kg}^{-1} \mathrm{BW}\right.$ day ${ }^{-1}$ ) & $383.33 \pm 3.170^{\mathrm{A}}$ & $307.67 \pm 2.900^{\mathrm{D}}$ & $346.00 \pm 2.640^{c}$ & $290.67 \pm 1.200^{\mathrm{E}}$ & $364.00 \pm 2.300^{B}$ \\
\hline \multicolumn{6}{|l|}{ Experiment 2} \\
\hline & $\mathrm{C} 2$ & PP1 & PP2 & PP3 & PP4 \\
\hline Dissolved oxygen (DO) $\mathrm{mg} \mathrm{L}^{-1}$ & $6.80 \pm 0.030^{A}$ & $6.38 \pm 0.080^{B}$ & $6.58 \pm 0.020^{A B}$ & $6.58 \pm 0.080^{\mathrm{AB}}$ & $6.53 \pm 0.040^{B}$ \\
\hline $\mathrm{pH}$ & $7.57 \pm 0.050^{\mathrm{A}}$ & $7.55 \pm 0.070^{\mathrm{A}}$ & $7.54 \pm 0.150^{A}$ & $7.60 \pm 0.090^{A}$ & $7.69 \pm 0.190^{A}$ \\
\hline Temperature $\left({ }^{\circ} \mathrm{C}\right)$ & $26.19 \pm 0.140^{\mathrm{A}}$ & $26.35 \pm 0.010^{A}$ & $26.43 \pm 0.050^{\mathrm{A}}$ & $26.41 \pm 0.170^{A}$ & $26.45 \pm 0.170^{\mathrm{A}}$ \\
\hline Conductivity ( $\mu$ mhos $\mathrm{cm}^{-1}$ ) & $653.83 \pm 3.170^{C}$ & $662.45 \pm 5.550^{\mathrm{ABC}}$ & $657.28 \pm 5.410^{B C}$ & $679.17 \pm 0.480^{\mathrm{A}}$ & $674.11 \pm 3.320^{\mathrm{AB}}$ \\
\hline Alkalinity $\left(\mathrm{mgL}^{-1}\right)$ & $189.33 \pm 1.200^{\mathrm{A}}$ & $186.22 \pm 1.920^{\mathrm{A}}$ & $187.45 \pm 0.390^{\mathrm{A}}$ & $188.67 \pm 1.890^{\mathrm{A}}$ & $190.67 \pm 2.220^{\mathrm{A}}$ \\
\hline Chloride $\left(\mathrm{mg} \mathrm{L}^{-1}\right)$ & $39.70 \pm 0.480^{\mathrm{A}}$ & $39.54 \pm 0.360^{A}$ & $39.20 \pm 0.190^{\mathrm{A}}$ & $39.59 \pm 0.250^{\mathrm{A}}$ & $39.70 \pm 0.240^{\mathrm{A}}$ \\
\hline Calcium $\left(\mathrm{mgL}^{-1}\right)$ & $19.10 \pm 0.410^{\mathrm{A}}$ & $18.92 \pm 0.500^{A}$ & $18.41 \pm 0.490^{\mathrm{A}}$ & $18.55 \pm 0.540^{\mathrm{A}}$ & $18.55 \pm 0.860^{\mathrm{A}}$ \\
\hline Hardness $\left(\mathrm{mgL}^{-1}\right)$ & $81.22 \pm 0.730^{\mathrm{A}}$ & $79.56 \pm 1.100^{\mathrm{A}}$ & $81.11 \pm 1.390^{\mathrm{A}}$ & $81.56 \pm 0.590^{A}$ & $80.56 \pm 2.350^{A}$ \\
\hline Total $\mathrm{NH}_{3}-\mathrm{N}$ excretion ( $\mathrm{mg} \mathrm{Kg}^{-1} \mathrm{BW}$ day $^{-1}$ ) & $676.33 \pm 2.330^{A}$ & $535 \pm 2.880^{B}$ & $439 \pm 2.300^{\mathrm{D}}$ & $363.33 \pm 2.030^{\mathrm{E}}$ & $445.33 \pm 2.910^{C}$ \\
\hline Total o- $\mathrm{PO}_{4}$ production $\left(\mathrm{mg} \mathrm{Kg}^{-1} \mathrm{BW}_{\text {day }}{ }^{-1}\right.$ ) & $344.67 \pm 1.45^{\mathrm{A}}$ & $295.33 \pm 1.45^{B}$ & $307 \pm 2.88^{C}$ & $206.67 \pm 1.76^{\mathrm{E}}$ & $289.33 \pm 2.33^{\mathrm{D}}$ \\
\hline
\end{tabular}

Note: All values are mean \pm S.E. of mean. Values with different superscript in the same row are significantly $(P<0.05)$ different (Tukey's honest test)

fingerlings depicted significant $(P<0.05)$ increase in comparison to other treatments and control.

\section{Phagocytic activity \\ Experiment 1}

The phagocytic activity and phagocytic indices of fish fed on a peppermint-supplemented diet after 90 days were significantly higher in treatment P3 (M. piperita @ $6 \mathrm{~g} \mathrm{Kg}^{-1}$ ) followed by P2 (M. piperita @ $\left.4 \mathrm{~g} \mathrm{Kg}^{-1}\right), \mathrm{P} 1$ (M. piperita@4 $\mathrm{g} \mathrm{Kg}^{-1}$ ), P4 (M. piperita@8 $\mathrm{g} \mathrm{Kg}^{-1}$ ), and control (Table 7).

\section{Experiment 2}

The phagocytic activity $(82.09 \pm 0.83)$ and phagocytic index $(2.96 \pm 0.01)$ of fingerlings fed on peppermint along with probiotics at $6 \mathrm{~g} \mathrm{Kg}^{-1}$ (treatment PP3) showed significant $(P<0.05)$ increase than all other treatments in the second experiment (Table 7).

\section{Respiratory burst activity \\ Experiment 1}

In this experiment, respiratory burst activity of treatment P3 $(0.84 \pm 0.01)$ followed by treatment P2 $(0.71 \pm$ $0.010)$, treatment $\mathrm{P} 4(0.63 \pm 0.01)$, and $\mathrm{P} 1(0.53 \pm 0.01)$ was significantly $(P<0.05)$ higher than control treatment $(0.48 \pm 0.01)$ (Table 7).

\section{Experiment 2}

Respiratory burst activity of fingerlings fed on different inclusion levels of peppermint with probiotics was analyzed in which treatment PP3-fed group fingerlings exhibited significantly $(P<0.05)$ higher values of respiratory burst activity in comparison with fingerlings of all other treatments in the second experiment (Table 7).

\section{Serum bactericidal activity Experiment 1}

Serum Bactericidal Activity is a measure of total bacterial colony count $\left(10^{3} \mathrm{CFU} \mathrm{ml}{ }^{-1}\right)$. The respective total 
Table 6 Proximate carcass composition of experimental diets containing M. piperita without or with probiotic bacterium $B$. coagulans (Experiment 1 and Experiment 2)

\begin{tabular}{|c|c|c|c|c|c|c|}
\hline \multirow{2}{*}{$\frac{\text { Proximate composition }}{\text { Experiment } 1}$} & \multicolumn{6}{|c|}{ Dietary treatments } \\
\hline & & & & & & \\
\hline & Initial value & $\mathrm{C} 1$ & P1 & P2 & P3 & P4 \\
\hline Crude protein (\%) & $9.27 \pm 0.020$ & $15.34 \pm 0.020^{\mathrm{D}}$ & $15.43 \pm 0.020^{C D}$ & $15.55 \pm 0.020^{B}$ & $15.93 \pm 0.030^{A}$ & $15.52 \pm 0.010^{B C}$ \\
\hline Crude fat (\%) & $4.03 \pm 0.020$ & $6.48 \pm 0.010^{\mathrm{D}}$ & $6.65 \pm 0.010^{C}$ & $6.76 \pm 0.010^{B}$ & $6.99 \pm 0.020^{A}$ & $6.81 \pm 0.030^{B}$ \\
\hline Total ash (\%) & $2.59 \pm 0.010$ & $3.67 \pm 0.010^{\mathrm{D}}$ & $3.82 \pm 0.020^{C}$ & $3.94 \pm 0.020^{B}$ & $4.17 \pm 0.010^{A}$ & $3.98 \pm 0.020^{B}$ \\
\hline Moisture (\%) & $64.76 \pm 0.030$ & $63.72 \pm 0.020^{\mathrm{D}}$ & $64.25 \pm 0.030^{C}$ & $64.25 \pm 0.080^{C}$ & $63.87 \pm 0.030^{C}$ & $64.47 \pm 0.030^{B}$ \\
\hline Nitrogen free extract (\%) & $21.94 \pm 0.040$ & $14.46 \pm 0.040^{\mathrm{A}}$ & $13.67 \pm 0.040^{B}$ & $13.43 \pm 0.070^{B C}$ & $12.21 \pm 0.010^{\mathrm{D}}$ & $13.20 \pm 0.050^{C}$ \\
\hline Gross energy $\left(\mathrm{kJ} \mathrm{g}^{-1}\right)$ & $7.54 \pm 0.020$ & $8.60 \pm 0.0020^{c}$ & $8.62 \pm 0.010^{B C}$ & $8.67 \pm 0.010^{B}$ & $8.79 \pm 0.004^{A}$ & $8.62 \pm 0.003^{B C}$ \\
\hline \multicolumn{7}{|l|}{ Experiment 2} \\
\hline & Initial value & $\mathrm{C} 2$ & PP1 & PP2 & PP3 & PP4 \\
\hline Crude protein (\%) & $9.27 \pm 0.020$ & $15.62 \pm 0.040^{\mathrm{D}}$ & $15.77 \pm 0.030^{C}$ & $15.95 \pm 0.020^{B}$ & $16.14 \pm 0.040^{A}$ & $15.79 \pm 0.020^{C}$ \\
\hline Crude fat (\%) & $4.03 \pm 0.020$ & $6.69 \pm 0.030^{C}$ & $6.82 \pm 0.030^{B}$ & $6.93 \pm 0.020^{A}$ & $7.00 \pm 0.030^{A}$ & $6.95 \pm 0.020^{A}$ \\
\hline Total ash (\%) & $2.59 \pm 0.010$ & $3.62 \pm 0.010^{C}$ & $3.59 \pm 0.020^{C}$ & $3.76 \pm 0.030^{B}$ & $3.93 \pm 0.020^{A}$ & $3.98 \pm 0.020^{A}$ \\
\hline Moisture (\%) & $64.76 \pm 0.030$ & $62.72 \pm 0.010^{\mathrm{D}}$ & $62.88 \pm 0.020^{C}$ & $63.10 \pm 0.030^{B}$ & $63.23 \pm 0.020^{A}$ & $62.94 \pm 0.020^{C}$ \\
\hline Nitrogen free extract (\%) & $21.94 \pm 0.040$ & $14.97 \pm 0.090^{\mathrm{A}}$ & $14.52 \pm 0.060^{B}$ & $14.02 \pm 0.050^{C}$ & $13.62 \pm 0.140^{D}$ & $14.31 \pm 0.020^{B}$ \\
\hline Gross energy $\left(\mathrm{kJ} \mathrm{g}^{-1}\right)$ & $7.54 \pm 0.020$ & $8.90 \pm 0.010^{A}$ & $8.92 \pm 0.003^{A}$ & $8.92 \pm 0.010^{A}$ & $8.94 \pm 0.010^{A}$ & $8.94 \pm 0.010^{A}$ \\
\hline
\end{tabular}

Note: All values are mean \pm S.E. of mean. Values with different superscript in the same row are significantly $(P<0.05)$ different $($ Tukey's honest test)

bacterial count of fish serum against pathogenic strain fed on different diets such as P1, P2, P3, and P4 are $4.88 \pm 0.05, \quad 4.62 \pm 0.03, \quad 4.03 \pm 0.15$, and $4.36 \pm 0.08$ $\left(10^{3} \mathrm{CFU} \mathrm{ml} \mathrm{m}^{-1}\right)$ indicating significantly improved activity in fingerlings of group P3. The value in the serum of fishes in the control (C1) was $5.10 \pm 0.08$ (Table 7).

\section{Experiment 2}

Serum Bactericidal Activity of fingerlings fed on peppermint at $6 \mathrm{~g} \mathrm{Kg}^{-1}$ along with probiotics showed significantly $(P<0.05)$ better response than other treatments in the second experiment as viable bacterial colony count is lowest compared to the rest of treatments including control (Table 7).

\section{Total serum protein}

\section{Experiment 1}

Total serum protein $\left(\mu \mathrm{g} \mathrm{ml}^{-1}\right)$ in this experiment was found significantly $(P<0.05)$ higher in the fingerlings fed on peppermint-supplemented diet at $6 \mathrm{~g} \mathrm{Kg}^{-1}(3.94 \pm$ $0.01)$ followed by $4 \mathrm{~g} \mathrm{Kg}^{-1}(3.59 \pm 0.02), 8 \mathrm{~g} \mathrm{Kg}^{-1}(3.51 \pm$ $0.01)$, and $2 \mathrm{~g} \mathrm{Kg}^{-1}(3.42 \pm 0.02)$ (Table 7).

\section{Experiment 2}

In this experiment, significantly $(P<0.05)$ higher total serum protein was detected in fingerlings fed on peppermint-supplemented diet along with probiotics at 6 $\mathrm{g} \mathrm{Kg}^{-1}$. The respective total serum protein values $(\mu \mathrm{g}$ $\mathrm{ml}^{-1}$ ) of fingerlings of different treatments such as P1, P2,
P3, and P4 are $3.42 \pm 0.02,3.59 \pm 0.02,3.94 \pm 0.01$, and $3.51 \pm 0.01$, and in the control the value is $3.38 \pm 0.01$.

\section{Relative percent survival after challenge trial Experiment 1}

Post challenge relative survival rates of fingerlings fed on peppermint-supplemented dietary treatment P3 $(67.93 \pm 6.62)$ were recognized significantly $(P<0.05)$ high in comparison to other dietary treatments such as P2 $(50.95 \pm 4.97)$ followed by P4 $(33.96 \pm 3.31)$ and P1 (16.98 \pm 1.65$)$, while the fingerlings fed on control diet showed the highest mortality $(60.00 \pm 5.77)$ and fingerlings of treatment P3 exhibited the lowest mortality $(20.00 \pm 5.77)$ (Fig. 2a). The Kaplan-Meier plots for survival of fish in Experiment 1 after challenge trial are presented in Fig. 3 a revealing that there is a significant difference between the survival trends in each treatment. The initial mortality was observed in treatment control, P1 and P4, while in treatment P2 and P3 mortality was observed after 3 days and 6 days, respectively.

\section{Experiment 2}

Post challenge relative survival rates of fingerlings fed on peppermint-supplemented dietary treatment along with probiotics at $6 \mathrm{~g} \mathrm{Kg}^{-1}(82.22 \pm 9.68)$ were examined significantly $(P<0.05)$ high in contrast with other dietary treatments such as PP2 $(56.11 \pm 16.95)$ followed by PP4 $(41.11 \pm 4.84)$ and PP1 $(20.55 \pm 2.42)$, while the fingerlings fed on the control diet revealed the highest 


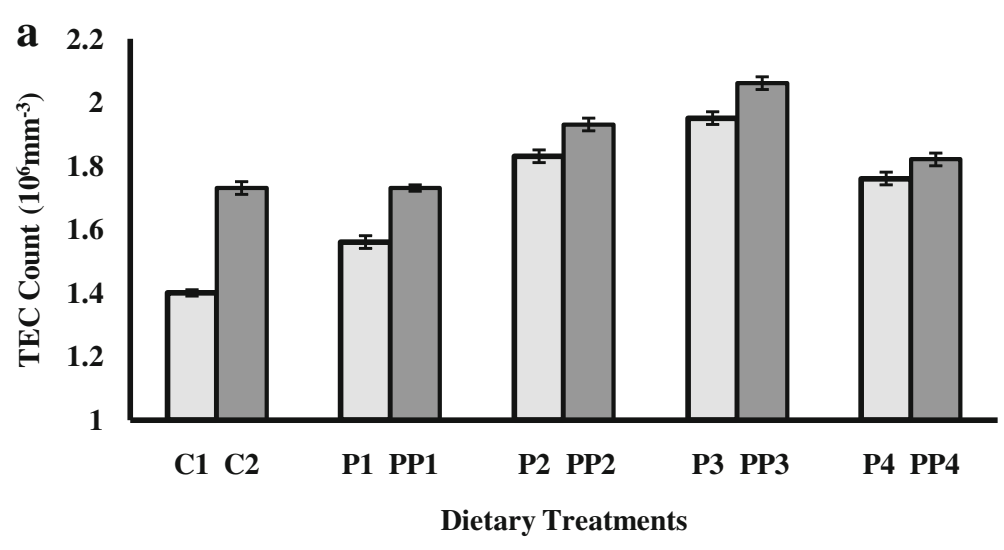

QWithout probiotic aWith Probiotic

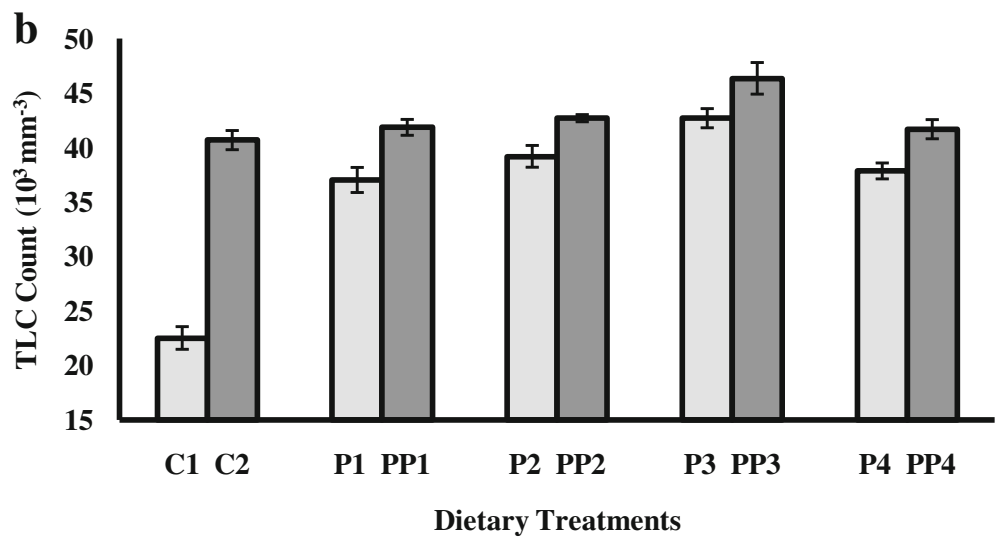

\section{QWithout Probiotic aWith Probiotic}

Fig. 1 Effect of M. piperita-supplemented diet without or with probiotic bacterium B. coagulans on hematological parameters (on total erythrocytes count (a) and on total leucocytes count (b)) of C. Catla. M. piperita-supplemented diet without probiotic bacterium B. coagulans (C1 $=$ Control, $\mathrm{P} 1=2 \mathrm{~g} \mathrm{Kg}^{-1}, \mathrm{P} 2=4 \mathrm{~g} \mathrm{Kg}^{-1}, \mathrm{P} 3=6 \mathrm{~g} \mathrm{Kg}^{-1}, \mathrm{P} 4=8 \mathrm{~g} \mathrm{Kg}^{-1}$ ) and $M$. piperita-supplemented diet with probiotic bacterium B. coagulans ( $C 2=$ Control, $\mathrm{PP} 1=2 \mathrm{~g} \mathrm{Kg}^{-1}, \mathrm{PP} 2=4 \mathrm{~g} \mathrm{Kg}^{-1}, \mathrm{PP} 3=6 \mathrm{~g} \mathrm{Kg}^{-1}$ and PP4 $=8 \mathrm{~g} \mathrm{Kg}^{-1}$ )

mortality $(50.00 \pm 5.77)$ and fingerlings of treatment PP3 exhibited the lowest mortality $(10.00 \pm 5.77)$ (Fig. 2b). The Kaplan-Meier plots for survival of fish in Experiment 2 after challenge trial are presented in Fig. $3 \mathrm{~b}$ revealing that there is a significant difference between the survival trends in each treatment. The initial mortality was observed in treatment control, P1 and P4, while in treatment PP2 and PP3 mortality was observed after 3 days and 7 days, respectively.

\section{Discussion}

Plant extracts have been reported to various prospective as a growth stimulator in aquatic creatures (Citarasu, Babu, Sekar, and Petermarian, 2002). The present study concedes that $C$. catla when fed on M. piperita-supplemented diets significantly $(P<0.05)$ increased the growth performance along with a better immune response. $M$. piperita has been acknowledged as appetite accelerator because it promotes daily feed consumption (Nobakht and Mehmannavaz, 2010), and subsequently, low values of FCR ultimately led to high weight gain, high growth \% in body weight, high SGR, high GCE in $M$. piperita-fed group fishes. The reason might be attributed that $M$. piperita could upgrade the digestibility and proper utilization of nutrition leading to protein synthesis which impacted ultimately on growth performance. The results in first experiment of our study are thus in accordance with the study of Talpur (2014) and Adel, Amiri, Zorriehzahra, Nematolahi, and Esteban (2015a) who fed M. piperita diets to Asian seabass, Lates calcarifer (Bloch), for 8 weeks and fry Caspian white fish for 4 weeks, respectively, and observed significantly higher growth rate in test fishes in comparison to control.

Crude protein and gross energy of fishes fed on a peppermint-supplemented diet at $6 \mathrm{~g} \mathrm{Kg}^{-1}$ (treatment P3) 
Table 7 Effect of fish fed on M. piperita-supplemented diet without or with probiotic bacterium B. coagulans on immunological parameters (Experiment 1 and Experiment 2)

\begin{tabular}{|c|c|c|c|c|c|}
\hline \multirow{2}{*}{$\frac{\text { Immunological parameters }}{\text { Experiment } 1}$} & \multicolumn{5}{|c|}{ Dietary treatments } \\
\hline & \multirow[b]{2}{*}{ C1 } & \multirow[b]{2}{*}{ P1 } & \multirow[b]{2}{*}{ P2 } & \multirow[b]{2}{*}{ P3 } & \multirow[b]{2}{*}{ P4 } \\
\hline & & & & & \\
\hline Phagocytic activity & $67.13 \pm 0.540^{C}$ & $71.73 \pm 0.890^{\mathrm{E}}$ & $75.40 \pm 0.740^{B}$ & $77.24 \pm 1.190^{A}$ & $67.96 \pm 1.260^{\circ}$ \\
\hline Phagocytic index & $1.43 \pm 0.003^{\mathrm{E}}$ & $1.83 \pm 0.010^{C}$ & $2.04 \pm 0.010^{B}$ & $2.54 \pm 0.003^{\mathrm{A}}$ & $1.64 \pm 0.040^{\mathrm{D}}$ \\
\hline Respiratory burst activity (NBT) & $0.48 \pm 0.010^{\mathrm{D}}$ & $0.53 \pm 0.010^{D}$ & $0.71 \pm 0.010^{B}$ & $0.84 \pm 0.010^{\mathrm{A}}$ & $0.63 \pm 0.010^{c}$ \\
\hline Serum bactericidal activity $\left(10^{3} \mathrm{CFU} \mathrm{ml}{ }^{-1}\right)$ & $5.10 \pm 0.080^{A}$ & $4.88 \pm 0.050^{A B}$ & $4.62 \pm 0.030^{B C}$ & $4.03 \pm 0.150^{\mathrm{D}}$ & $4.37 \pm 0.080^{C D}$ \\
\hline Serum protein $\left(\mu \mathrm{g} \mathrm{m}^{-1}\right)$ & $3.38 \pm 0.010^{c}$ & $3.42 \pm 0.020^{C}$ & $3.59 \pm 0.020^{B}$ & $3.94 \pm 0.010^{\mathrm{A}}$ & $3.51 \pm 0.010^{B}$ \\
\hline \multicolumn{6}{|l|}{ Experiment 2} \\
\hline & $\mathrm{C} 2$ & PP1 & PP2 & PP3 & PP4 \\
\hline Phagocytic activity & $73.52 \pm 1.280^{\mathrm{D}}$ & $71.65 \pm 0.590^{\mathrm{E}}$ & $76.43 \pm 0.150^{B}$ & $82.09 \pm 0.830^{A}$ & $74.26 \pm 0.980^{c}$ \\
\hline Phagocytic index & $1.92 \pm 0.010^{\mathrm{D}}$ & $2.11 \pm 0.010^{C}$ & $2.19 \pm 0.010^{B}$ & $2.96 \pm 0.010^{A}$ & $1.77 \pm 0.010^{\mathrm{E}}$ \\
\hline Respiratory burst activity (NBT) & $0.80 \pm 0.010^{c}$ & $0.83 \pm 0.010^{C}$ & $0.89 \pm 0.010^{B}$ & $1.02 \pm 0.010^{\mathrm{A}}$ & $0.90 \pm 0.010^{B}$ \\
\hline Serum bactericidal activity $\left(10^{3} \mathrm{CFU} \mathrm{ml}{ }^{-1}\right)$ & $4.75 \pm 0.030^{A}$ & $4.40 \pm 0.050^{B}$ & $3.80 \pm 0.050^{C}$ & $3.43 \pm 0.040^{\mathrm{D}}$ & $3.81 \pm 0.030^{C}$ \\
\hline Serum protein $\left(\mu \mathrm{g} \mathrm{ml}^{-1}\right)$ & $3.55 \pm 0.010^{\mathrm{E}}$ & $3.81 \pm 0.010^{C}$ & $3.92 \pm 0.010^{B}$ & $4.17 \pm 0.010^{A}$ & $3.70 \pm 0.010^{\mathrm{D}}$ \\
\hline
\end{tabular}

Note: All values are mean \pm S.E. of mean. Values with different superscript in the same row are significantly $(P<0.05)$ different $($ Tukey's honest test)

were found significantly high when compared with other treatments. The NFE in test fish may illustrate that they do not accumulate carbohydrate in their tissue as a major part was used as a reservoir of energy. The NFE content recognized during proximate analysis might be developed from basic sources such as glycoproteins and glycolipids. Our results for NFE content in fishes during proximate analysis are in thus accordance with the findings by Bob-Manuel and Alfred-Ockiy (2011) and Orire and Sadiku (2014) in which they observed NFE (\%) content in the range of $16.33-38.11$ and $0.17-8.66$, respectively. Peppermint promotes the synthesis of protein in high amounts and thus high protein accumulation. Enzymatic activity results of the present study concluded that peppermint can upgrade intestinal enzymes and microbiota of fish. Significant differences were observed in enzymatic activities (protease, amylase, cellulose) of treated groups as compared to control. On the contrary, no significant $(P<0.05)$ difference was observed in protease activity but significant $(P<0.05)$ difference was recorded for amylase activity of Caspian brown trout when they were fed on a peppermint-supplemented diet by Adel, Safari, Pourgholam, Zorriehzahra, and Esteban (2015b). The reason might be due to different feeding habits of Caspian brown trout in their study and C. catla in the present study. As vegetable protein is difficult to digest in non-carnivorous fish, it requires more proteolytic enzyme activity for utilizing the protein present in the diet. In our study, total leucocytes and total erythrocytes count were also increased significantly $(P<0.05)$ in $M$. piperita-supplemented diet-fed group which is similar to the findings of Adel et al. (2015 a, b) and Nya and Austin (2009) who obtained increased levels of $\mathrm{RBC}$ and $\mathrm{WBC}$ when Caspian brown trout were fed on different peppermintsupplemented diets and Oncorhynchus mykiss fed on different ginger-supplemented diets. However, post challenge data reveal that WBC count of blood increased and RBC count of blood decreased, as the increase of WBC count is an important phenomenon of defense when any foreign microorganism causes any infection. Our results coincide with the results of Talpur and Ikhwanuddin (2012) and of Talpur, Ikhwanuddin, and Bolong (2013) who also realized the increase count of WBC when L. calcifer were fed on garlic- and gingersupplemented diet, and Talpur (2014) determined similar results when $L$. calcifer were fed on different $M$. piperita-supplemented diets.

Phagocytosis and respiratory burst activity is acknowledged as a predominant mechanism countering bacterial defense. Mentha species have phenomenal antimicrobial and antioxidant properties as reported by Mimica-Dukic, Bozin, Sokovic, Mihajlovic, and Matavulj (2003) and Mahboubi and Haghi (2008). The result of the present study manifested high phagocytic activity (Table 7) in peppermint-supplemented diet which clearly revealed that supplementation of $M$. piperita in diet enhanced the nonspecific immune response significantly $(P<0.05)$ which is related to the results of Abasali and Mohamad (2010) and Talpur (2014) who reported the remarkably increased activity of phagocytosis in C. carpio and Lates calcifer after feeding on $M$. piperita-supplemented diets. The superoxide anion production can be assessed by Nitroblue 

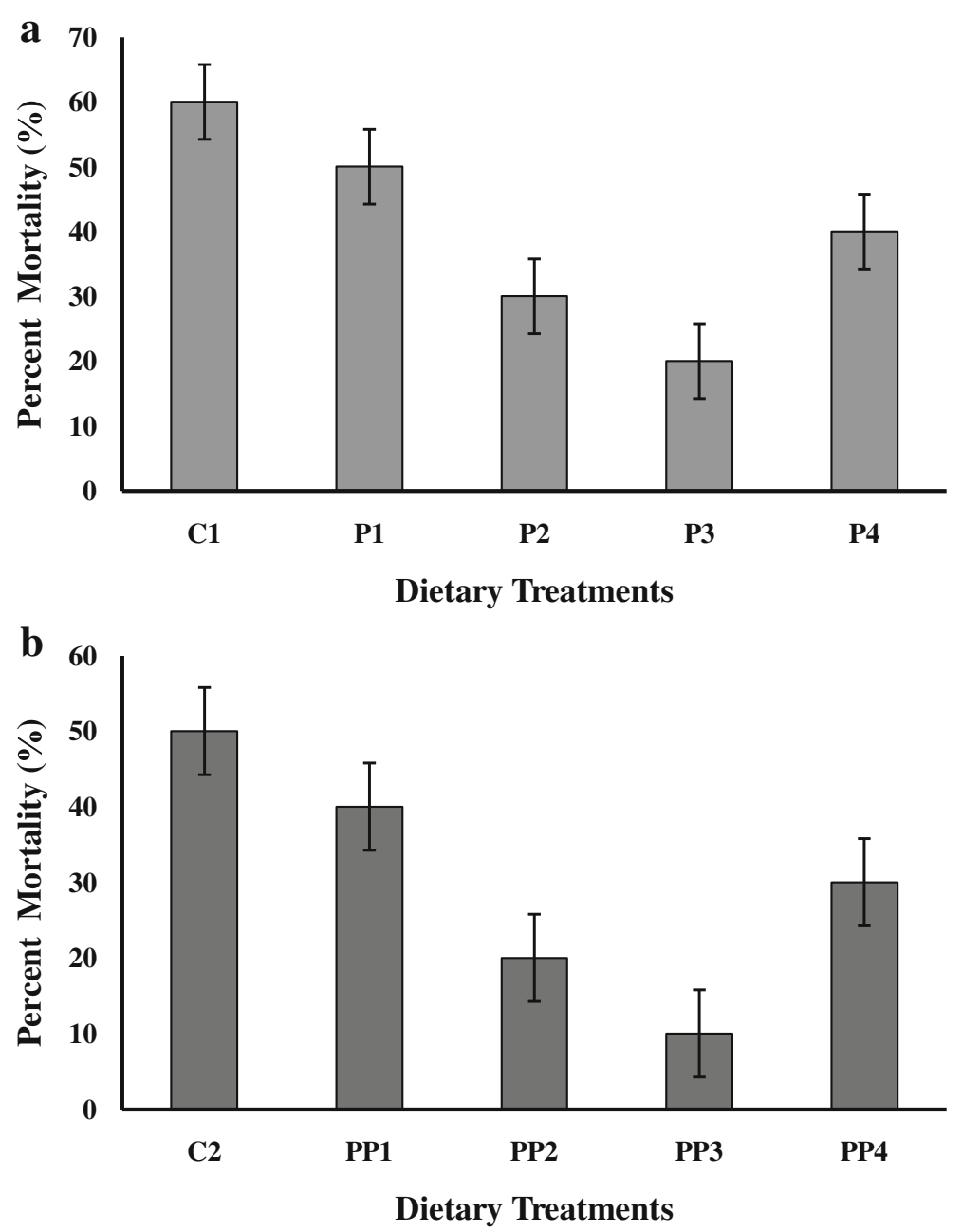

Fig. 2 Percent mortality of C. Catla fed on M. piperita-supplemented diet without (a) or with (b) probiotic bacterium B. coagulans (Experiment 1 and Experiment 2). M. piperita-supplemented diet without probiotic bacterium B. coagulans ( $\mathrm{C} 1=$ Control, $\mathrm{P} 1=2 \mathrm{~g} \mathrm{Kg}^{-1}, \mathrm{P} 2=4 \mathrm{~g} \mathrm{Kg}$, $\mathrm{P} 3=6$ $\mathrm{g} \mathrm{Kg}^{-1}, \mathrm{P} 4=8 \mathrm{~g} \mathrm{Kg}^{-1}$ ) and $M$. piperita-supplemented diet with probiotic bacterium B. coagulans ( $\mathrm{C} 2=$ Control, $\mathrm{PP} 1=2 \mathrm{~g} \mathrm{Kg}^{-1}, \mathrm{PP} 2=4 \mathrm{~g} \mathrm{Kg}{ }^{-1}$, PP3 $=6 \mathrm{~g} \mathrm{Kg}^{-1}$, and PP4 $=8 \mathrm{~g} \mathrm{Kg}^{-1}$ )

tetrazolium assay (NBT) by calibrating free oxygen radicals (Anderson and Siwicki, 1995). The outcome of the present study illustrated that respiratory burst activity was observed to be significantly $(P<0.05)$ higher in treatment P3 fed group fingerlings containing $M$. piperita at $6 \mathrm{~g} \mathrm{Kg}^{-1}$ of feed.

A crucial immune response which is concerned with the conquering of pathogenic microbes is serum bactericidal activity (Ellis, 1999). Serum bactericidal activity of all treated fed group fingerlings showed significant increase than the control treatment. Remarkably, better serum bactericidal activity was observed in treatment P3 (containing peppermint $6 \mathrm{~g} \mathrm{Kg}^{-1}$ of feed), as pathogenic bacteria plate count was found to be lesser in this treatment in comparison to others. Our results are constant with the results of Nya and Austin (2009) who achieved elevated serum bactericidal activity when Oncorhynchus mykiss were fed on ginger-supplemented diets; Abasali and Mohamad (2010) who calculated the bacterial count and observed the increased serum bactericidal activity after feeding on M. piperita in C. carpio; Talpur and Ikhwanuddin (2012), Talpur et al. (2013), and Talpur and Ikhwanuddin (2013), who assessed the increased serum bactericidal activity after feeding on garlic, neem leaf and ginger, respectively, in L. calcifer; and Talpur (2014) who found the significantly $(P<0.05)$ high serum bactericidal activity in L. calcifer after feeding on $M$. piperita. This improved serum bactericidal activity in these studies and also in the present study clearly reveal better immune response which might be attributable to the existence of bioactive components such as phenol, tannins, and flavanoids (Pramila et al., 2012) in M. piperita leaves which are known for antimicrobial and antiinflammatory properties (de Sousa Araújo, Alencar, de 

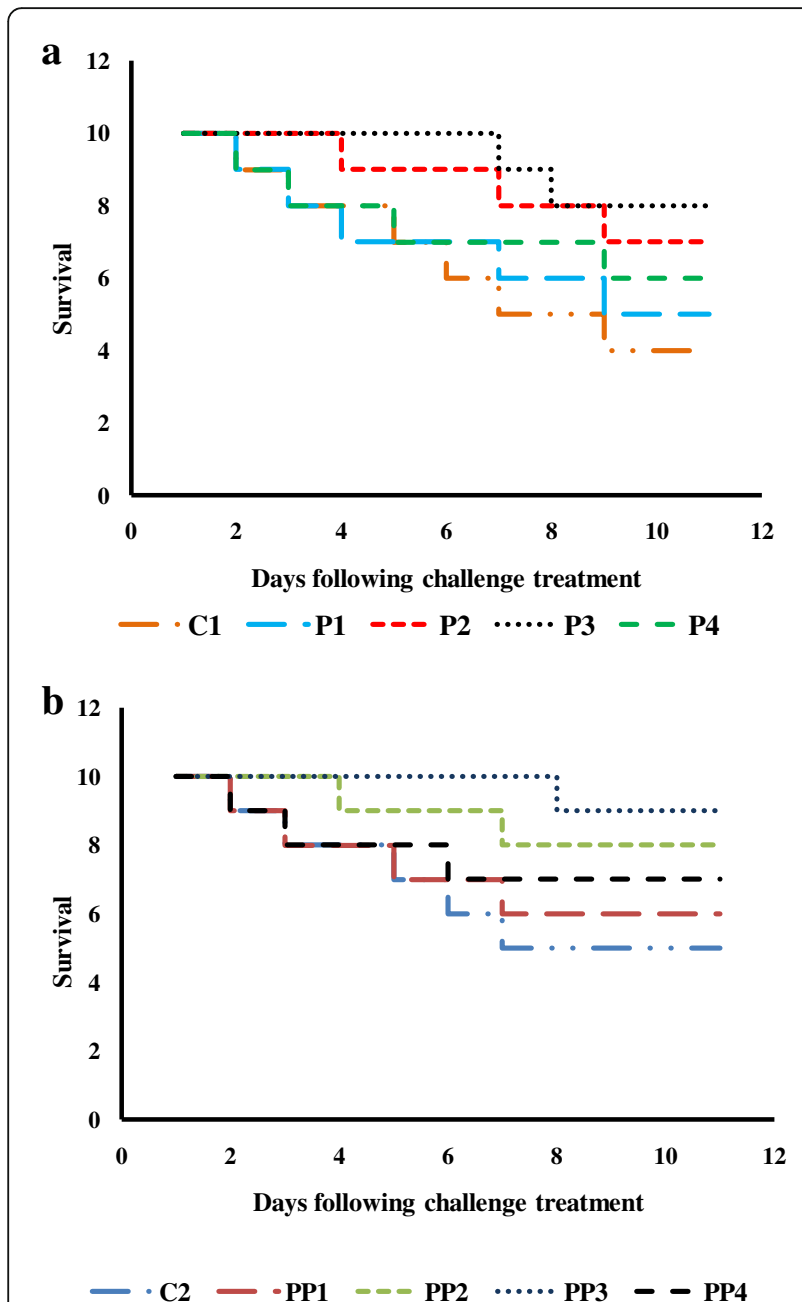

Fig. 3 Plots of Kaplan-Meier estimates of survival of C. Catla after challenge trial in different M. piperita-supplemented diets without (a) or with (b) probiotic bacterium B. coagulans (Experiment 1 and Experiment 2). M. piperita-supplemented diet without probiotic bacterium B. coagulans ( $\mathrm{C} 1=$ Control, $\mathrm{P} 1=2 \mathrm{~g} \mathrm{Kg}^{-1}, \mathrm{P} 2=4 \mathrm{~g} \mathrm{Kg}^{-1}$, $\mathrm{P} 3=6 \mathrm{~g} \mathrm{Kg}^{-1}, \mathrm{P} 4=8 \mathrm{~g} \mathrm{Kg}^{-1}$ ) and $M$. piperita-supplemented diet with probiotic bacterium B. coagulans ( $C 2=$ Control, $P P 1=2 \mathrm{~g} \mathrm{Kg}^{-1}$, $\mathrm{PP} 2=4 \mathrm{~g} \mathrm{Kg}^{-1}, \mathrm{PP} 3=6 \mathrm{~g} \mathrm{Kg}^{-1}$, and PP4 $=8 \mathrm{~g} \mathrm{Kg}^{-1}$ )

Amorim, and de Albuquerque, 2008). The serum protein level was also monitored and significantly $(P<0.05)$ higher values were observed in fingerlings fed on $M$. piperita at $6 \mathrm{~g} \mathrm{Kg}^{-1}$ than their respective control treatment fingerlings which resembled with the results of Sunitha et al. (2017) who observed significant increase in serum protein in Cyprinus carpio after feeding on Phyllanthus niruri. The values of serum protein reflect the capability of the liver for protein synthesis and signify renal functioning and blood osmolarity (Sunitha et al., 2017). Thus, high serum protein in treatment P3 clearly reveals better capability of protein synthesis and thus coinciding with high growth and high carcass protein; therefore, supplementation of $M$. piperita at $6 \mathrm{~g} \mathrm{Kg}^{-1}$ appears to be optimum dose. Moreover, increasing level of serum protein in the group of fish fed on $M$. piperita-supplemented diets might be due to active components such as menthone, isomenthone, and 1, 8-cineole (Mimica-Dukic et al., 2003), which helps in the prevention of different infectious diseases leading to better immunity.

In the second experiment, probiotic bacterium Bacillus coagulans at $3000 \mathrm{CFU} \mathrm{g}^{-1}$ of feed was also incorporated along with different levels of $M$. piperita. The result showed high growth performance in terms of WG, SGR, PER, GCE, and APD with low FCR in group of fishes fed on diet PP3 (with M. piperita @ $6 \mathrm{~g} \mathrm{Kg}^{-1}$ of feed and $B$. coagulans@3000 CFU g ${ }^{-1}$ ). WBC and RBC count was significantly high along with high phagocytic activity, respiratory burst activity, and better serum bactericidal activity with low excretion of metabolites (ammonia excretion and reactive phosphate production) in group of fingerlings of treatment PP3 which further demonstrated that inclusion of $M$. piperita @ $6 \mathrm{~g} \mathrm{Kg}^{-1}$ of diet is optimum for better growth performance, nutrient retention, and immunity of C. catla. On comparing the results of Experiment 1 and 2, it was observed that the growth performance, nutrient retention, and immunity parameter values were high for the respective groups of Experiment 2 with significantly higher value in treatment PP3 where fishes were fed on a diet containing M. piperita @ 6 $\mathrm{g} \mathrm{Kg}^{-1}$ of diet and B. coagulans @ $3000 \mathrm{CFU} \mathrm{g}^{-1}$. This might be attributed to the reason that the presence of autochthonous probiotic bacterium supplemented in diet altered the microbial metabolism of fishes or might be due to the colonization of probiotic bacteria in the gut of fishes that expanded its exogenous enzyme production ultimately promoting the growth performance (Bhatnagar et al., 2012). It has been accounted that probiotic bacterium also provides the unsaturated fatty acid and vitamins involved in host nutrition (Sakata, 1990).

On comparison with TLC and TEC count in Experiment 1 and 2, TLC and TEC count increased with increasing dose of $M$. piperita up to the level of $6 \mathrm{~g} \mathrm{Kg}^{-1}$ of diet and then count declined with further increasing the dose in the first experiment. It clearly revealed that M. piperita-supplemented diet @ $6 \mathrm{~g} \mathrm{Kg}^{-1}$ of feed is optimum. However, in Experiment 2, the same trend was observed, but with the addition of probiotic along with $M$. piperita-supplemented diet, TLC and TEC count were increased more at each respective dose level (Fig. 1). This might be due to the reason that active compounds present in $M$. piperita and probiotic bacterium synergistically stimulated the humoral immunity at optimum level. Probiotic bacterium incorporated in diet inflected an immune response of fishes by depressing the pathogenic responses by enhancing leucocyte count which involved in phagocytic activity. Balcazar (2003) illustrated the incorporation of several probiotic 
bacterium strain (Bacillus and Vibrios sp.), decidedly impacted the development and survival of adolescents of white shrimp, and displayed a defensive impact against Vibrio harveyi because of an incitement of immune system by expanding phagocytic activity and antiviral activity. Moreover, Nikoskelainen, Ouwehand, Bylund, and Salminen (2003) demonstrated that supplementation of Lactobacillus rhamnosus @ of $10^{5} \mathrm{CFU} \mathrm{g}^{-1}$ of feed accelerated the respiratory burst activity in Oncorhynchus mykiss. Significant high phagocytic activity, phagocytic index, respiratory burst activity, and better serum bactericidal activity of probiotic-supplemented fingerlings in our study also supported this view as it enhanced the production of phagocytes during invasive attack of pathogen by defense mechanism. In addition to this, supplementation of $M$. piperita in the diet also improved feed consumption expanding the intestinal activity with proper utilization of feed which resulted in synergistic effects on growth performance and immunomodulatory responses in Catla catla. The challenge trial acknowledged that oral administration of $M$. piperita-supplemented diet along with probiotic bacterium boosted the defiance of C. catla from pathogenic infection and coincided with the results of Bhatnagar and Lamba (2017) in which B. cereus was when incorporated in diets at different inclusion level for Cirrhinus mrigala showed the high survival of probiotic-fed group fishes as compared to the control diet fishes.

The Kaplan-Meier assessment is the simplest way to figure out the survival over time despite all these complications related with subjects or circumstances. This survival pattern clearly indicated that treatment P3 and PP3 showed high survival while high mortality was detected in fingerlings of control treatment in both experiments conceding the immunostimulating effects of dietary supplementation of $M$. piperita as well as of probiotics.

\section{Conclusion}

The results of the present study signified that use of $M$. piperita-supplemented diet can significantly improve growth and immunity in C. catla without compromising water quality, and further improvement can be obtained when such diets are further supplemented with autochthonous probiotic bacterium. Since, to the knowledge of authors, no work has been conducted yet on the synergistic effect of autochthonous probiotic bacterium- and plant-supplemented diet, so it can make a better approach towards detailing of an ideal diet by using supplementation of different plant extract along with other autochthonous probiotics for better growth performance and immunity of fish that can prompt sustainable aquaculture.

\begin{abstract}
Abbreviations
@: at the rate of; <: is less than; \pm : plus-minus; ANOVA: Analysis of variance; APD: Apparent Protein Digestibility; APHA: American Public Health Organization; BW: Body weight; CFU: Colony forming unit; DO: Dissolved oxygen; EDTA: Ethylenediaminetetraacetic acid; FAO: Food and Agricultural Organization; FCR: Feed conversion ratio; GCE: Gross conversion efficiency;

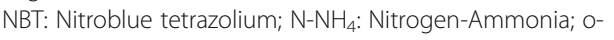

$\mathrm{PO}_{4}$ : Orthophosphate; PA: Phagocytic activity; PER: Protein efficiency ratio; PI: Phagocytic index; RBC: Red blood cells; RPS: Relative percent survival; SGR: Specific growth rate; SPSS: Statistical Package for Social Sciences; t: Time; TEC: Total erythrocyte count; TLC: Total leucocyte count; TSA: Tryptone soya agar; W: Weight; WBC: White blood cells; WG: Weight Gain
\end{abstract}

\section{Acknowledgement}

SS is sincerely thankful to HSCST-JRF (HSCST-2301) for fellowship.

\section{Authors' contributions}

$A B$ designed the experiment and participated in the preparation of the manuscript. SS carried out the experiment and analyzed the samples/data. Both authors read and approved the final manuscript.

\section{Funding}

SS is sincerely thankful to HSCST-JRF (HSCST-2301) for fellowship.

\section{Availability of data and materials}

All data sets generated and/or analyzed during the current study are available with the corresponding author

\section{Ethics approval}

Ethical guidelines for the use of animal care were followed

\section{Consent for publication}

Not applicable

\section{Competing interests}

The authors declare that they have no competing interests.

Received: 2 April 2019 Accepted: 15 July 2019

Published online: 30 July 2019

\section{References}

Abasali H, Mohamad S. Immune response of common carp (Cyprinus carpio) fed with herbal iminunostimulants diets. J Anim Vet Adv. 2010;9(13):1839-47.

Adel M, Amiri AA, Zorriehzahra J, Nematolahi A, Esteban MÁ. Effects of dietary peppermint (Mentha piperita) on growth performance, chemical body composition and hematological and immune parameters of fry Caspian white fish (Rutilus frisii kutum). Fish Shellfish Immunol. 2015a;45(2):841-7. https://doi.org/10.1016/j.fsi.2015.06.010

Adel M, Safari R, Pourgholam R, Zorriehzahra J, Esteban MÁ. Dietary peppermint (Mentha piperita) extracts promote growth performance and increase the main humoral immune parameters (both at mucosal and systemic level) of Caspian brown trout (Salmo trutta caspius Kessler, 1877). Fish Shellfish Immunol. 2015b;47(1):623-9. https://doi.org/10.1016/j.fsi.2015.10.005.

Anderson DP, Siwicki AK. Basic haematology and serology for fish health programs. In: Shariff M, Arthur JR, Subasinghe RP, editors. Diseases in Asian Aquaculture II. Manila: Philippines Fish Health Section, Asian Fisheries Society; 1995. p. 185-202.

AOAC (Association of Official Analytical Chemists). Official methods of analysis. Arlington: Association of Official Analytical Chemists Incorporation; 1995. p. 684.

APHA (American Public Health Association). Standard methods for the examination of water and waste water. 20th ed. New York: American Public Health Association; 1998.

Austin B, Stuckey LF, Robertson PAW, Effendi I, Griffith DRW. A probiotic strain of Vibrio alginolyticus effective in reducing diseases caused by Aeromonas salmonicida, Vibrio anguillarum and Vibrio ordalii. J Fish Dis. 1995;18(1):93-6. https://doi.org/10.1111/j.1365-2761.1995.tb01271.x.

Balcazar JL. Evaluation of probiotic bacterial strains in Litopenaeus vannamei. Guayaquil: Final Report, National Center for Marine and Aquaculture Research; 2003. 
Balcazar JL, Blas D, Zarzuela IR, Vendrell D, Girones O, Muzquiz JL. Sequencing of variable regions of the $16 \mathrm{~S}$ rRNA gene for identification of lactic acid bacteria isolated from the intestinal microbiota of healthy salmonids. Comp Immunol Microbiol Infect Dis. 2007;30:111-8. https://doi.org/10.1016/j.cimid.2006.12.001.

Bandyopadhyay P. Improvement of growth, nutritional quality and immunity of Indian major carps through probiotic supplement. Midnapore, West Bengal: Ph.D. Thesis, Vidyasagar University; 2004.

Bhatnagar A, Lamba R. Molecular characterization and dosage application of autochthonous potential probiotic bacteria in Cirrhinus mrigala. J Fisheries Sci Com. 2017;11(2):46-56. https://doi.org/10.21767/1307-234X.1000117.

Bhatnagar A, Raparia S. Optimum dietary inclusion level of Bacillus coagulans for growth and digestibility improvement for Catla catla (Hamilton). Int J Curr Res Rev. 2014;6(7):1-10.

Bhatnagar A, Raparia S, Kumari S. Isolation and influence of Bacillus coagulans CC1 on growth performance and digestive enzyme activities of Catla catla (Hamilton). J Nat Sci Sustainable Technol. 2012;6(3):237-53.

Blumenthal M. The complete German commission E monographs: Therapeutic guide to herbal medicines. Austin: American botanical council; 1998.

Bob-Manuel FG, Alfred-Ockiya JF. Evaluation of yeast single cell protein (SCP) diets on growth performance, feed conversion and carcass composition of Tilapia Oreochromis niloticus (L.) fingerlings. African J Biotechnol. 2011; 10(46):9473-7478.

Cho CY, Slinger SJ, Bayley HS. Bioenergetics of salmonid fishes: Energy intake, expenditure and productivity. Comp Biochem Physiol. 1982;73(1):25-41. https://doi.org/10.1016/0305-0491(82)90198-5.

Citarasu T, Babu MM, Sekar RRJ, Petermarian M. Developing Artemia enriched herbal diet for producing quality larvae in Penaeus monodon, Fabricius. Asian Fisheries Sci. 2002;15(1):21-32.

Dacie JV, Lewis SH. Practical Hematology. $3^{\text {rd }}$ ed: London: J and A Churchill Ltd; 1963.

de Sousa Araújo TA, Alencar NL, de Amorim ELC, de Albuquerque UP. A new approach to study medicinal plants with tannins and flavonoids contents from the local knowledge. J Ethnopharmacol. 2008;120(1):72-80. https://doi. org/10.1016/j.jep.2008.07.032.

Ellis AE. Fish vaccination. London: Academic Press; 1988. p. 84.

Ellis AE. Immunity to bacteria in fish. Fish Shellfish Immunol. 1999;9(4):291-308. https://doi.org/10.1006/fsim.1998.0192

FAO/WHO. Health and nutritional properties of probiotics in food including powder milk with live lactic acid bacteria. Report of a joint FAO/WHO expert consultation on evaluation of health and nutritional properties of probiotics in food including powder milk with live lactic acid bacteria. Cordoba: FAO and WHO Publication. 2001;1-34.

Garg SK, Bhatnagar A, Kalla A, Johal MS. Experimental ichthyology. New Delhi: CBS Publications and Distributors; 2002.

Ghosh S, Sinha A, Sahu C. Isolation of putative probionts from the intestines of Indian major carps. Israeli J Aquaculture - Bamidgeh. 2007;59(3):127-32.

Gornall AG, Bardawill CS, David MM. J Biol and Chem. 1949;177:751-3.

Kajita Y, Sakai M, Atsuta S, Kobayashi M. The immunomodulatory effects of levamisole on rainbow trout, Oncorhyncus mykiss. Fish Pathology. 1990;25: 93-8. https://doi.org/10.3147/jsfp.25.93.

Kaplan EL, Meier P. Nonparametric estimation from incomplete observations. Journal of the American statistical association. 1958;53(282):457-481.

Mahboubi M, Haghi G. Antimicrobial activity and chemical composition of Mentha pulegium L. essential oil. J Ethnopharmacol. 2008;19:325-7. https:// doi.org/10.1016/j.jep.2008.07.023

Mimica-Dukic N, Bozin B, Sokovic M, Mihajlovic B, Matavulj M. Antimicrobial and antioxidant activities of three Mentha species essential oils. Planta Medica. 2003;69:413-9. https://doi.org/10.1055/s-2003-39704.

Nayak SK, Mukherjee SC. Screening of gastrointestinal bacteria of Indian major carps. Aquaculture Res. 2011;42:1034-41. https://doi.org/10.1111/j.1365-2109.2 010.02686.x.

Nikoskelainen S, Ouwehand AC, Bylund G, Salminen S, Lilius EM. Immune enhancement in rainbow trout (Oncorhynchus mykiss) by potential probiotic bacteria (Lactobacillus rhamnosus). Fish Shellfish Immunol. 2003;15(5):443-52. https://doi.org/10.1016/S1050-4648(03)00023-8.

Nobakht A, Mehmannavaz Y. Investigation the effects of using of Thymus vulgaris, Lamiaceae menthapiperita, Oreganum valgare medicinal plants on performance, egg quality, blood and immunity parameters of laying hens. Iranian J Appl Anim Sci. 2010;41:129-36.

Nya E, Austin B. Use of dietary ginger, Zingiber officinale Roscoe as an immunostimulant to control Aeromonas hydrophila infections in rainbow trout, Oncorhynchus mykiss (walbaum). J Fish Dis. 2009:32:971-7. https://doi.org/10.1111/j.1365-2761.2009.01101.x.
Orire AM, Sadiku SO. Effects of carbohydrate sources on the growth and body compositions of African catfish (Clarias gariepinus). Int J Fisheries Aquaculture. 2014;6(5):55-61.

Park $\mathrm{KH}$, Jeong $\mathrm{HD}$. Enhanced resistance against Edwardsiella tarda infection in tilapia Oreochromis niloticus by administration of protein bound polysaccharide. Aquaculture. 1996;141:135-43. https://doi.org/10.1016/ 0044-8486(95)01224-9.

Pramila DM, Xavier R, Marimuthu K, Kathiresan S, Khoo ML, Senthilkumar M, Sathya K, Sreeramanan S. Phytochemical analysis and antimicrobial potential of methanolic leaf extract of peppermint (Mentha piperita: Lamiaceae). J Med Plants Res. 2012;6(2):331-5. https://doi.org/10.5897/JMPR11.1232.

Quesada SP, Paschoal JAR, Reyes FGR. Considerations on the aquaculture development and on the use of veterinary drugs: special issue for fluoroquinolones_a review. J Food Sci. 2013;78(9):1321-33. https://doi.org/1 $0.1111 / 1750-3841.12222$.

Rico A, Phu TM, Satapornvanit K, Min J, Shahabuddin AM, Henriksson PJG, Murray FJ, Little DC, Dalsgaard A, Van den Brink PJ. Use of veterinary medicines, feed additives and probiotics in four major internationally traded aquaculture species farmed in Asia. Aquaculture. 2013;412:231-43. https://doi.org/10.1 016/j.aquaculture.2013.07.028.

Sadasivam S, Manickam A. Biochemical methods 2nd edn. New Delhi: New Age International Publishers (P) Ltd.; 1996. p. 107-9.

Sahoo SK, De TK, Ghosh PK, Maitra A. pH and thermo-sensitive hydrogel nanoparticles. J Colloid Interface Sci. 1998;206(2):361-8. https://doi.org/10.1 006/jcis.1998.5692.

Sakata T. Microflora in the digestive tract of fish and shellfish. In: Lesel $R$, editor. Microbiology in Poecilotherms. Amsterdam: Elsevier; 1990. p. 171-6.

Sawhney SK, Singh R. Introductory Practical Biochemistry. New Delhi: Narosa Publishing House; 2000. pp. 452.

Seyfried EE, Newton RJ, Rubert KF, Pedersen JA, McMahon KD. Occurrence of tetracycline resistance genes in aquaculture. Facilities with varying use of oxytetracycline. Microbial Ecology. 2010;59:799-807. https://doi.org/10.1007/ s00248-009-9624-7.

Siwicki AK, Anderson DP, Rumsey GL. Dietary intake of immunostimulants by rainbow trout affects non-specific immunity and protection against furuncuclosis. Vet Immunol Immunopathol. 1994;41:125-39. https://doi.org/1 0.1016/0165-2427(94)90062-0.

Sunitha C, Mettilda S, Vinoliya J. Effect of dietary intake of Phyllanthus niruri L. on fingerlings of freshwater fish, Cyprinus carpio L. Int J Fisheries Aquatic Studie. 2017:5(1):352-9.

Sushma. Effect of artificial feeding and probiotics on growth performance, survival and digestibility in fresh water fish, Aorichthys aor (Hamilton). Kurukshetra, Haryana: M.Phil. Dissertation, Kurukshetra University; 2007.

Talpur AD. Mentha piperita (peppermint) as feed additive enhanced growth performance, survival, immune response and disease resistance of Asian seabass, Lates calcarifer (Bloch) against Vibrio harveyi infection. Aquaculture. 2014:420:71-8. https://doi.org/10.1016/j.aquaculture.2013.10.039.

Talpur AD, Ikhwanuddin M. Dietary effects of garlic (Allium sativum) on haematoimmunological parameters, survival, growth, and disease resistance against Vibrio harveyi infection in Asian sea bass, Lates calcarifer (Bloch). Aquaculture. 2012;364:6-12. https://doi.org/10.1016/j.aquaculture.2012.07.035.

Talpur AD, Ikhwanuddin M. Azadirachta indica (neem) leaf dietary effects on the immunity response and disease resistance of Asian seabass, Lates calcarifer challenged with Vibrio harveyi. Fish Shellfish Immunol. 2013;34(1):254-64. https://doi.org/10.1016/j.fsi.2012.11.003.

Talpur AD, Ikhwanuddin M, Bolong AMA. Nutritional effects of ginger (Zingiber officinale Roscoe) on immune response of Asian sea bass, Lates calcarifer (Bloch) and disease resistance against Vibrio harveyi. Aquaculture. 2013;400: 46-52. https://doi.org/10.1016/j.aquaculture.2013.02.043.

Tukey JW. Some thoughts on clinical trials, especially problems of multiplicity. Science. 1977;198(4318):679-84.

Walter HE. Probionases: Methods with haemoglobin, casein and azocoll as substrates. In: Bergmeyer HU, editor. Methods of Enzymatic Analysis, Vol. V. Weinheim: Verlag Chemic; 1984.

\section{Publisher's Note}

Springer Nature remains neutral with regard to jurisdictional claims in published maps and institutional affiliations. 